\title{
Normas brasileiras para o Affective Norms for English Words
}

\author{
Brazilian norms for the Affective Norms for English Words
}

\author{
Christian Haag Kristensen ${ }^{1}$, Carlos Falcão de Azevedo Gomes ${ }^{2}$, Alice Reuwsaat Justo ${ }^{3}$, Karin Vieira ${ }^{4}$
}

\section{Resumo}

Introdução: O presente estudo realizou a tradução e adaptação do Affective Norms for English Words (ANEW) para o português brasileiro (ANEW-Br) e obteve medidas de emocionalidade para um conjunto de 1.046 palavras em português.

Método: Uma amostra de 755 universitários de ambos os sexos utilizou as escalas de valência e alerta do Self-Assessment Manikin para julgar a emocionalidade de 1.046 palavras em português.

Resultados: Os valores de valência ficaram no intervalo de 1,16 a 8,80 , enquanto que os valores de alerta ficaram no intervalo de 2,22 a 7,67. Análises adicionais indicaram que as medidas de valência e alerta se mostraram fidedignas, além de sugerir adequação do método utilizado para coleta das medidas de emocionalidade.

Conclusão: A disponibilização das normas brasileiras para o ANEW representa um avanço metodológico para pesquisadores brasileiros no desenvolvimento de futuros estudos sobre os efeitos da emoção na cognição humana.

Descritores: Emoções, alerta, cognição.

\begin{abstract}
Introduction: The present study translated and adapted the Affective Norms for English Words (ANEW) to Brazilian Portuguese (ANEW-Br) and collected emotionality measures for a set of 1,046 words in Brazilian Portuguese.

Method: A sample of 755 male and female undergraduate students used the valence and arousal scales of the Self-Assessment Manikin to judge the emotionality of 1,046 words in Brazilian Portuguese.

Results: Valence values ranged from 1.16 to 8.80 , while arousal values ranged from 2.22 to 7.67 . Further analyses indicated that both valence and arousal measures were reliable and suggested that the method used was appropriate for the collection of emotionality measures.

Conclusion: The availability of Brazilian norms for the ANEW represents a methodological advancement for Brazilian investigators in the development of future studies about the effects of emotion on human cognition.
\end{abstract}

Keywords: Emotions, arousal, cognition.

\section{Introdução}

O estudo científico das emoções pode ser datado na segunda metade do século XIX, com os trabalhos seminais de G. B. Duchenne de Boulogne e Charles Darwin, seguidos pelas contribuições de William James e Walter B. Cannon. Embora diversificadas, as abordagens desses autores possuíam, em comum, o emprego da experimentação e da observação sistemática na busca de

definições conceituais das emoções, bem como sua funcionalidade e estrutura ${ }^{1,2}$. No entanto, ao longo da maior parte do século $X X$, o estudo das emoções foi relegado ao segundo plano. Em parte, tal situação foi gerada por uma falta de consenso sobre a natureza e a estrutura das emoções, bem como sobre os procedimentos metodológicos para acessá-las e mensurá-las³.

\footnotetext{
${ }^{1}$ Doutor. Professor adjunto, Pontifícia Universidade Católica do Rio Grande do Sul (PUCRS), Porto Alegre, RS. ${ }^{2}$ Acadêmico de Psicologia, PUCRS. Bolsista de Iniciação Científica, Conselho Nacional de Desenvolvimento Científico e Tecnológico (CNPq). ${ }^{3}$ Acadêmico de Psicologia, PUCRS. Bolsista de Iniciação Científica, PUCRS. ${ }^{4}$ Psicóloga. Trainee em Neuropsicologia, Hospital São Lucas, PUCRS.

Apoio financeiro: Fundação de Amparo à Pesquisa do Estado do Rio Grande do Sul (FAPERGS), Universidade do Vale do Rio dos Sinos (UNISINOS; protocolo no 02.00.008/03-0) e Conselho Nacional de Desenvolvimento Científico e Tecnológico (CNPq; protocolo nº 104185/2010-4).

Recebido em 25/03/2011. Aceito em 02/04/2011. Não foram declarados conflitos de interesse associados à publicação deste artigo.

Como citar: Kristensen CH, Gomes CFA, Justo AR, Vieira K. Normas brasileiras para o Affective Norms for English Words. Trends Psychiatry Psychother. $2011 ; 33(3): 135-46$.
} 
Há pelo menos duas décadas, a emergência e a consolidação de disciplinas como a neurociência cognitiva ${ }^{4}$, com derivações teóricas, como a neurociência afetiva ${ }^{5,6}$, reposicionaram o estudo das emoções no centro da investigação científica7,8. Crucial para isso foi o desenvolvimento de estímulos padronizados para o estudo do processamento emocional. Em particular, os pesquisadores do Center for the Study of Emotion and Attention (CSEA), do National Institute of Mental Health (NIMH), na University of Florida, destacam-se pela produção de um conjunto de estímulos emocionais padronizados: International Affective Picture System (IAPS) ${ }^{9,10}$, International Affective Digital Sounds (IADS) ${ }^{11}$, Affective Norms for English Text (ANET) ${ }^{12}$ e Affective Norms for English Words (ANEW) ${ }^{13}$.

A teoria conceitual subjacente aos estímulos propostos por esse grupo sugere que a emoção evoluiu a partir de uma reação reflexa para responder a dois tipos básicos de estímulos: apetitivos ou aversivos ${ }^{14}$. Esses dois sistemas motivacionais podem variar em termos de alerta (arousal, em inglês), que representaria a intensidade metabólica ou neural da ativação tanto do sistema apetitivo quanto do sistema aversivo - ou mesmo a ativação conjunta desses dois sistemas. Em termos conceituais, a emoção pode ser definida como uma reação temporal breve de prontidão para ação, sendo composta por ao menos duas dimensões ortogonais, uma de valência (do desagradável ao agradável) e outra de alerta (do relaxado ao estimulado), em uma concepção definida como a teoria dimensional da emoção $0^{3,15,16}$. Dessa forma, é possível caracterizar um estímulo (por exemplo, a palavra "podre") no que diz respeito ao quão desagradável ou agradável o percebemos (isto é, a valência do estímulo) e ao quão relaxado ou estimulado ficamos perante ele (isto é, o alerta do estímulo). Por exemplo, estímulos que evocam uma emoção discreta de raiva podem ser classificados como de valência desagradável e alerta alto; estímulos que evocam uma emoção discreta de tristeza podem ser classificados como de valência desagradável e alerta baixo; estímulos que evocam uma emoção discreta de felicidade podem ser classificados como de valência agradável e alerta alto.

O conjunto de estímulos desenvolvidos pelo CSEA fundamentado na teoria dimensional das emoções - tem sido amplamente empregado em múltiplas linhas de investigação, envolvendo pesquisa básica ${ }^{17,18}$ e aplicada ${ }^{19,20}$ sobre emoções, bem como sobre o esforço de translação entre as mesmas ${ }^{21}$. Nessa perspectiva translacional, podem ser elencados diferentes tópicos relacionados ao estudo das emoções, incluindo mecanismos de regulação das emoções ${ }^{22,23}$. O estudo da regulação normal das emoções pode ser contrastado com a crescente ênfase sobre os efeitos que os distúrbios no processamento das emoções apresentam para o desenvolvimento e manutenção de psicopatologias ${ }^{1,24}$. Gradualmente, proliferam modelos que conceitualizam transtornos ansiosos e depressivos como resultados de problemas na regulação das emoções ${ }^{25,26}$. O avanço desses modelos depende, ao menos em parte, de paradigmas experimentais válidos e confiáveis. Logo, torna-se premente a disponibilização de estímulos padronizados para a investigação empírica da emoção.

Apesar da ampla utilização dos estímulos emocionais desenvolvidos no CSEA, identificamos, no Brasil, apenas a adaptação e normatização do IAPS ${ }^{27-29}$. No entanto, diversas tarefas experimentais necessitam da utilização de estímulos não pictóricos, como palavras (por exemplo, tarefas de aprendizagem de estímulos verbais, compreensão semântica e decisão lexical). Em língua inglesa, o ANEW ${ }^{13}$ encontra-se entre os conjuntos de palavras emocionais mais utilizados para investigação dos efeitos da emoção na cognição ${ }^{30-32}$. O ANEW consiste em um conjunto de 1.034 palavras com medidas para três dimensões emocionais. A primeira dimensão, chamada de valência (valence, em inglês), consiste na avaliação do quão agradável ou desagradável um estímulo é percebido. A segunda dimensão, chamada de alerta, consiste na avaliação do quão estimulado ou relaxado um estímulo nos deixa. A terceira dimensão, chamada de dominância (dominance, em inglês), consiste na avaliação do quão em controle de um estímulo ou dominado por ele nós nos percebemos.

O ANEW tem se mostrado uma ferramenta relevante na pesquisa sobre emoção, inspirando sua adaptação completa ou parcial em países como França ${ }^{33,34}$, Alemanha ${ }^{35,36}$, Espanha ${ }^{37}$, Finlândia e Inglaterra ${ }^{38}$. Em uma perspectiva translacional, palavras emocionais têm sido usadas, por exemplo, em estudos de memória episódica com amostras clínicas, como pacientes com doença de Alzheimer ${ }^{39}$ ou esquizofrenia ${ }^{40}$, e amostras não clínicas ${ }^{41}$. Considerando sua utilidade e relevância, o presente trabalho traduziu, adaptou e normatizou uma versão em português do ANEW para a população brasileira, adotando método equivalente ao estudo original realizado por Bradley \& Lang ${ }^{13}$. Especificamente, o objetivo do presente estudo foi obter medidas de valência e alerta para um conjunto de 1.046 palavras em português, caracterizando um estudo de tradução, adaptação e normatização do ANEW. Assim sendo, a publicação destas normas vem ao encontro dos trabalhos de normatização com palavras associadas $^{42,43}$ e figuras emocionais ${ }^{27-29}$ recentemente publicados, ao fornecer uma fonte a partir da qual pesquisadores brasileiros possam selecionar estímulos com características emocionais específicas e investigar os efeitos da emoção na cognição. 


\section{Método}

\section{Amostra}

Participaram deste estudo 755 universitários de cursos de ciências humanas e exatas de universidades públicas e privadas localizadas na região metropolitana de Porto Alegre (RS). A idade média dos participantes foi de 22 anos (desvio padrão, DP $=3$ anos), sendo que $73 \%$ eram do sexo feminino. Apenas participaram do estudo os estudantes cuja língua materna era o português brasileiro. O processo de amostragem foi feito por conveniência.

\section{Instrumentos}

Para avaliação da emocionalidade do conjunto final de 1.046 palavras em português, foi utilizada a escala Self-Assessment Manikin (SAM). Essa escala é composta por 9 pontos, sendo que 5 desses pontos são ancorados por figuras de bonecos que indicam graus de reações emocionais (Figura 1). Dessa forma, cada dimensão emocional possui uma escala própria, em que figuras de bonecos ilustram variações na definição para aquela dimensão. Por exemplo, o SAM que representa valência (Figura 1A) varia de uma figura sorridente (agradável) a uma figura com face descontente (desagradável); o SAM que representa alerta (Figura 1B) varia de uma figura ativada (estimulado) a uma figura inerte (relaxado).

A opção de utilizar o SAM como escala de avaliação emocional foi baseada em suas propriedades psicométricas e na tentativa de manter o método equivalente ao do estudo original?. Estudos de correlatos psicofisiológicos indicam que a) julgamentos de alerta estão associados a variações na condutância elétrica da pele, ao passo que b) julgamentos de valência estão associados a variações na contração de músculos faciais (notadamente os músculos zigomático maior e corrugador), medida através de eletromiografia facial ${ }^{44,45}$. Além disso, estudos de neuroimagem por ressonância magnética funcional sugerem que o processamento do alerta de um estímulo está associado à ativação da amígdala, enquanto o processamento da valência de um estímulo está associado à ativação de sub-regiões do córtex orbitofrontal ${ }^{46}$. Em conjunto, esses resultados indicam que o SAM possui boas características psicométricas e apontam para a adequação da teoria dimensional da emoção.

\section{Procedimentos}

O estudo foi dividido em duas grandes etapas: a) uma etapa de tradução e adaptação das palavras do ANEW $^{13}$ para o português brasileiro; e b) uma etapa de julgamento de valência e alerta para as palavras resultantes (ANEW-Br). Na primeira etapa, um linguista traduziu as 1.034 palavras do ANEW para o português brasileiro (tradução direta). Cada uma das palavras foi então novamente traduzida para o inglês (tradução reversa) por pelo menos dois pesquisadores com fluência na língua, obtendo-se duas listas de 1.034 palavras: uma em português por tradução direta e outra em inglês por tradução reversa. Posteriormente, um grupo de juízes independentes verificou a adequação das palavras traduzidas para o português brasileiro, utilizando como critério a equivalência semântica entre as duas traduções. Essa tarefa gerou concordância entre os juízes para $94,4 \%$ das traduções. Após esse processo, foram removidas todas as traduções que resultaram em palavras compostas, e novas palavras emocionais foram adicionadas, de forma que a lista final totalizou 1.046 palavras no idioma português brasileiro.

Na segunda etapa, a de julgamento da emocionalidade das 1.046 palavras, foram organizados 12 conjuntos

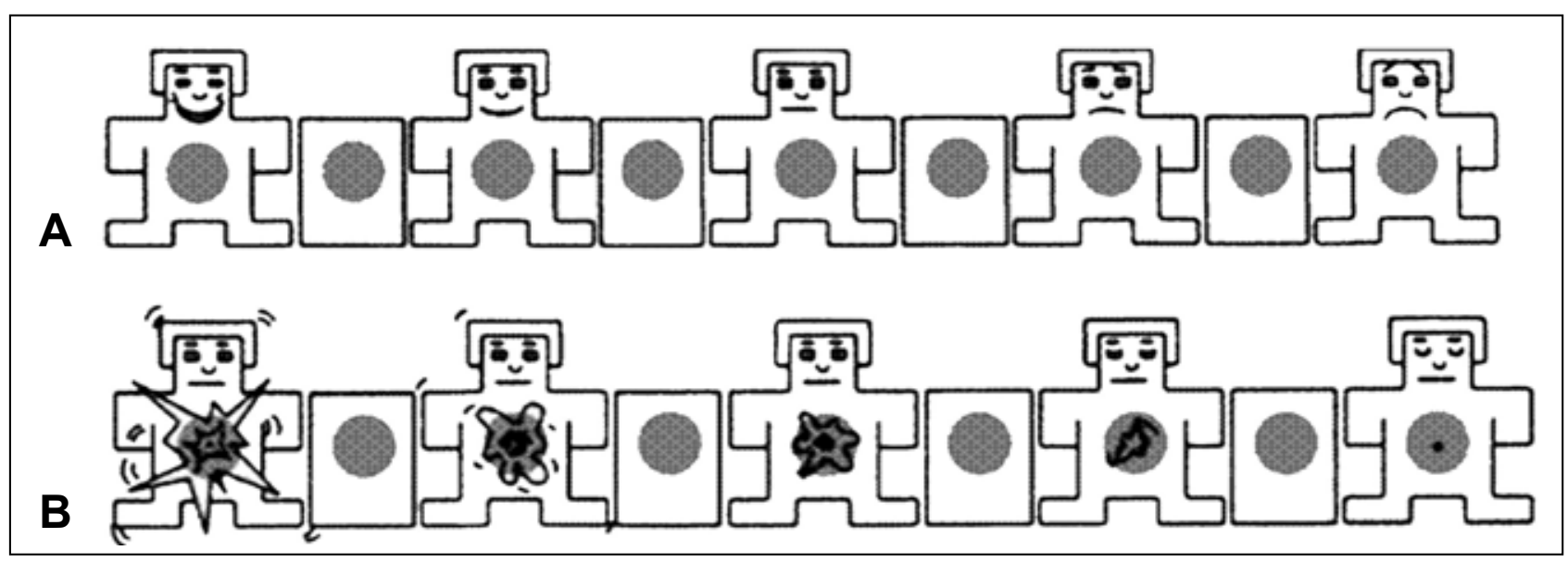

Figura 1 - Escala de avaliação de valência (A) e alerta (B) do Self-Assessment Manikin ${ }^{13}$ 
de palavras: 10 contendo 96 palavras e dois contendo 43 palavras. Essa divisão das 1.046 palavras foi adotada de forma que a tarefa dos participantes não fosse desgastante a ponto de influenciar os julgamentos de emocionalidade. Além disso, para evitar algum efeito da ordem de apresentação das palavras no julgamento de emocionalidade, cada conjunto teve duas ordens diferentes de apresentação (ordem A e ordem B). Assim, cada grupo de participantes avaliou no mínimo 43 e no máximo 96 palavras.

As sessões para o julgamento de emocionalidade foram realizadas em grupos. Antes do início de cada sessão, os participantes foram informados sobre o caráter de participação anônima e voluntária, sendo que apenas participaram aqueles que assinaram o termo de consentimento livre e esclarecido. Para aqueles que concordaram em participar da pesquisa, o pesquisador distribuiu um bloco com aproximadamente cinco folhas, sendo que cada folha continha 20 vocábulos e duas escalas SAM para cada vocábulo (uma para o julgamento de valência e a outra para o julgamento de alerta). Para o julgamento de valência, o pesquisador instruiu os participantes a marcarem com um " $X$ " o setor da escala de 9 pontos que correspondesse ao quão agradável (mais próximo ao boneco feliz; valor 9) ou desagradável (mais próximo ao boneco infeliz; valor 1 ) eles percebiam a palavra que foi apresentada. Para o julgamento de alerta, o pesquisador instruiu os participantes a marcarem com um " $X$ " o setor da escala de 9 pontos que correspondesse ao quão relaxado (mais próximo ao boneco calmo; valor 1 ) ou estimulado (mais próximo ao boneco ativado; valor 9) eles se sentiam perante a palavra que foi apresentada. Para ambas as escalas, o pesquisador enfatizou que os participantes poderiam marcar tanto os intervalos entre cada boneco quanto o ponto intermediário da escala (valor 5) caso a palavra fosse neutra na definição em questão (por exemplo, xícara é uma palavra que pode ser percebida como sendo nem agradável nem desagradável, isto é, neutra; nesse caso, o participante deveria marcar o ponto intermediário da escala de valência). Além disso, antes do início da tarefa, o pesquisador forneceu diversos exemplos para ilustrar como os julgamentos de emocionalidade deveriam ser feitos.

Após o esclarecimento de todas as dúvidas dos participantes, o pesquisador solicitou que eles começassem a tarefa de julgamento de emocionalidade. Ao término de todo o procedimento, o pesquisador agradeceu a participação de todos.

\section{Tratamento dos dados}

Para cada uma das 1.046 palavras, a análise descritiva envolveu computar médias e desvios padrão dos julgamentos de valência e alerta com base em uma amostra de no mínimo 38 e no máximo 80 participantes. Foram empregadas análises correlacionais para verificar possível efeito da ordem de apresentação das palavras nos índices de emocionalidade coletados. Finalmente, foram calculadas equações lineares e quadráticas para verificar o ajuste da distribuição das palavras do ANEW-Br em um espaço afetivo. Para todos os testes estatísticos, foi adotado a =0,05 como critério de significância.

\section{Resultados}

O número de caracteres de cada uma das 1.046 palavras que fizeram parte do ANEW-Br ficou no intervalo de 2 (por exemplo, ar, pé) até 14 caracteres (por exemplo, desinteressado, tranquilamente), com média de 7 caracteres ( $D P=2,05$ caracteres). No que se refere à valência emocional, os valores observados ficaram no intervalo de 1,16 (assassino) até 8,80 (liberdade), com média de 5,16 $(D P=2,20)$. No que se refere ao alerta emocional, os valores observados ficaram no intervalo de 2,22 (tranquilo) até 7,67 (assalto), com média de 4,57 ( $D P=1,04$ ). O Anexo 1 apresenta as médias e DP obtidos para as dimensões de valência e alerta em todas as palavras do ANEW-Br.

Foram calculadas correlações de Pearson entre as ordens de apresentação A e B dos conjuntos de palavras com o objetivo de verificar a influência da ordem de apresentação nos julgamentos de valência e alerta. Especificamente, esperava-se que, quanto menor a influência da ordem de apresentação e maior a fidedignidade das medidas de valência e alerta, maior seria a correlação direta entre as ordens A e B. Essa análise mostrou correlações diretas entre ordem $A$ e ordem $B$ tanto para valência $(r=0,963 ; \mathrm{p}<0,001)$ quanto para alerta $(r=0,685 ; \mathrm{p}<0,001)$. Portanto, as correlações sugerem duas conclusões sobre o ANEW-Br: primeiro, que a ordem de apresentação não parece ter influenciado os julgamentos de emocionalidade; segundo, que as medidas de valência e alerta se mostraram fidedignas.

A representação gráfica da percepção emocional para - ANEW-Br pode ser feita através da construção de um espaço afetivo ${ }^{16}$, no qual a valência ocupa o eixo das ordenadas e o alerta ocupa o eixo das abscissas. Na Figura 2 são apresentados os espaços afetivos referentes ao ANEW-Br e ao ANEW original (ANEW-USA). Como se pode observar, o espaço afetivo do ANEW-Br parece apresentar a mesma forma característica de "bumerangue" que o ANEW-USA. Para investigar essa observação, foram ajustadas a) uma equação linear e b) uma equação quadrática sob o espaço afetivo invertido da Figura 2 , no qual a valência é representada por $x$ e o alerta por y. Se o espaço afetivo do ANEW-Br segue um formato de 


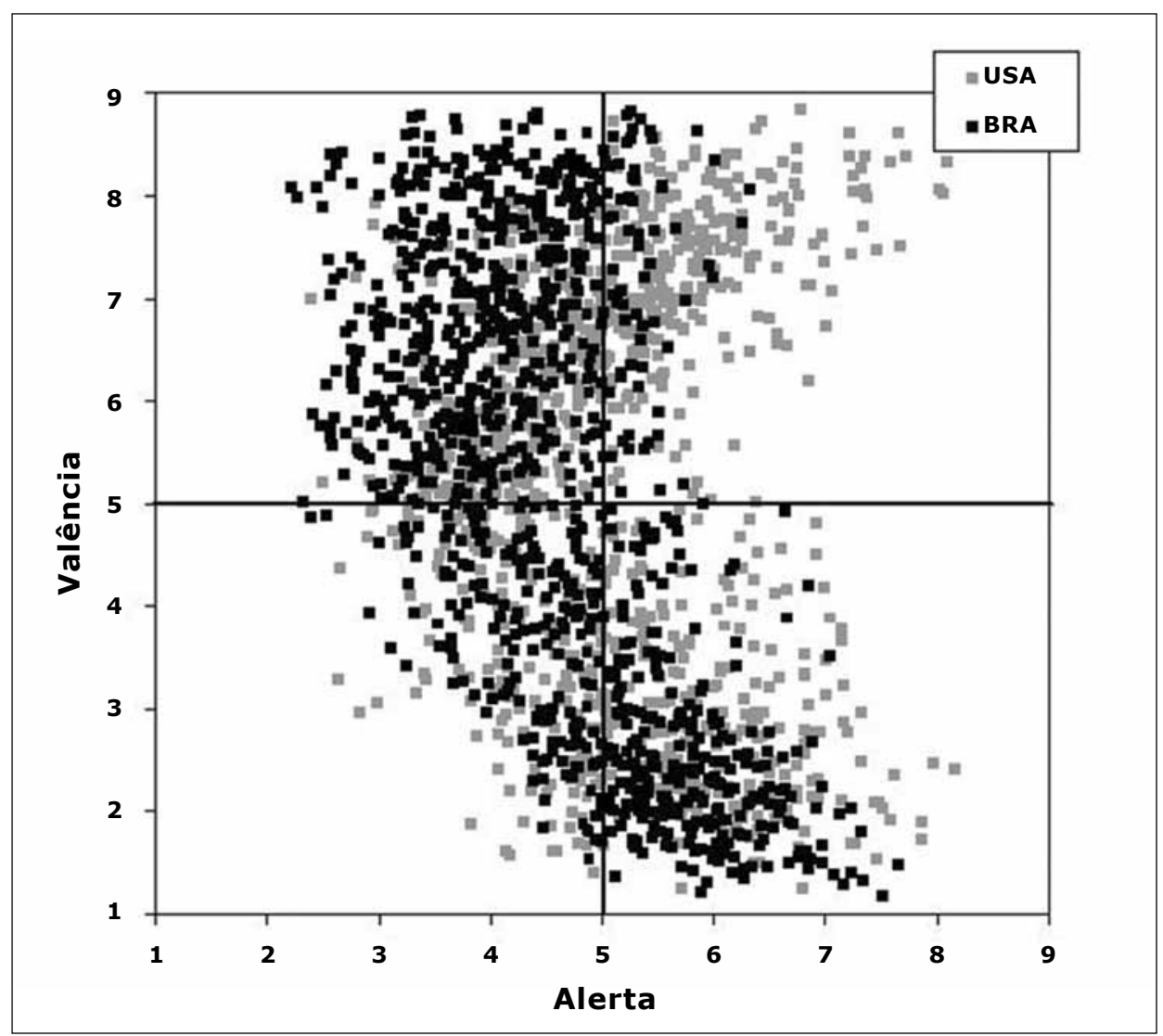

Figura 2 - Gráfico de dispersão das 1.046 palavras da versão brasileira do Affective Norms for English Words (BRA) e das 1.034 palavras do instrumento original (USA) segundo valência e alerta

bumerangue, então se espera que uma equação quadrática se ajuste melhor a esse conjunto de dados do que uma equação linear. De fato, uma equação quadrática explica $48 \%$ das variações no espaço afetivo do ANEW$-\operatorname{Br}\left(y=0,09 x^{2}-1,2 x+7,78 ; r=0,69 ; p<0,001\right)$, enquanto que uma equação linear explica $35 \%$ dessas variações ( $y=6,01-0,28 x ; r=0,59 ; p<0,001$ ).

$O$ espaço afetivo na Figura 2 também indica que nem todos os quadrantes são ocupados pelo mesmo número de palavras. Por exemplo, palavras desagradáveis (valência $<4$ ), como assassino, miséria e tortura, tendem a se concentrar no quadrante de alerta alto (alerta $>5$ ). Palavras agradáveis (valência $>6$ ), como abraço, mãe e amigo, tendem a se concentrar no quadrante de alerta baixo (alerta $<5$ ). Palavras neutras $(4<$ valência $<6$ ), como reunião, passagem e martelo, tendem a se concentrar nos quadrantes de alerta baixo.

\section{Discussão}

O objetivo principal do presente estudo foi obter medidas de emocionalidade para um conjunto de 1.046 palavras no idioma português brasileiro e disponibilizá-las à comunidade científica. Para atingir esse objetivo, foram coletadas, descritas e disponibilizadas as medidas de emocionalidade que compõem o ANEW-Br. A semelhança entre o espaço afetivo obtido com o ANEW-Br e aquele observado no ANEW americano sugere a adequação do método utilizado no presente estudo. Para somar a esse argumento, um formato similar da distribuição dos valores de valência e alerta observado no ANEW-Br também foi observado nas adaptações feitas em língua alemã ${ }^{36}$ e espanhola ${ }^{37}$.

A disponibilização das normas brasileiras para o ANEW representa um ganho metodológico no desenvolvimento de futuras pesquisas sobre os efeitos da emoção na cognição humana realizadas em solo nacional. Em países de língua inglesa, o ANEW, bem como outros materiais desenvolvidos pelo CSEA, tem sido utilizado na investigação básica ${ }^{17,18}$ e aplicada ${ }^{19,20}$ sobre a influência das emoções na cognição. Por exemplo, em amostras não clínicas, o desempenho da memória para palavras emocionais (agradáveis ou desagradáveis) é melhor do que para palavras neutras ${ }^{47,48}$. No entanto, pacientes com doença de Alzheimer usualmente não mostram meIhor desempenho da memória para palavras emocionais 
do que para neutras ${ }^{39}$, sugerindo que regiões neurais associadas com memória para informações emocionais (por exemplo, amígdala ${ }^{49}$ ) são afetadas pela doença.

Por fim, é importante ressaltar diferenças entre as normas brasileiras e as americanas, bem como uma limitação das normas brasileiras. Primeiro, o ANEW-Br não constitui apenas a tradução e normatização do ANEW. O ANEW-Br também é uma adaptação das normas americanas do ANEW. Devido à inadequação de traduções do inglês para o português e do português para o inglês (traduções ambíguas ou que resultaram em palavras compostas), diversas palavras que constam no ANEW não foram incluídas no conjunto de 1.046 palavras do ANEW-Br, e vice-versa. Segundo, assim como as normas americanas do ANEW ${ }^{13}$, as normas brasileiras foram obtidas através de avaliações de universitários e, portanto, devem ser utilizadas com essa ressalva. No entanto, diferentemente das normas americanas do ANEW, as normas brasileiras foram obtidas com universitários de cursos variados das áreas de exatas e humanas, e não apenas de cursos introdutórios de psicologia.

\section{Agradecimentos}

Os autores gostariam de agradecer a Adriane Nogueira, Emília Saatkamp, Larissa Weber, Marília Silveira, Renata Kochhann, Maria Alice de Mattos Pimenta Parente e Rosa Maria Martins de Almeida pela inestimável contribuição na coleta de dados e tradução das palavras.

Os autores também agradecem à Universidade do Vale do Rio dos Sinos, que apoiou financeiramente o projeto de pesquisa relacionado ao desenvolvimento do ANEW-Br.

\section{Referências}

1. Mayne TJ, Bonanno GA. Emotions: current issues and future directions. New York: Guilford Press; 2001.

2. Dalgleish T. The emotional brain. Nat Rev Neurosci. 2004;5:583-9.

3. Mauss IB, Robinson MD. Measures of emotion: a review. Cogn Emot. 2009;23:209-37.

4. Gazzaniga MS. The cognitive neurosciences. Cambridge: MIT; 1995.

5. Davidson RJ, Sutton SK. Affective neuroscience: the emergence of a discipline. Curr Opin Neurobiol. 1995;5:217-24.

6. Panksepp J. A critical role for 'affective neuroscience' in resolving what is basic about emotions. Psychol Rev. 1992;99:554-60.

7. Gazzaniga MS, Ivry RB, Mangun GR. Neurociência cognitiva: a biologia da mente. Porto Alegre: Artmed; 2006.

8. Marshall PJ. Relating psychology and neuroscience: taking up the challenges. Perspect Psychol Sci. 2009;4:113-25.

9. Lang PJ, Bradley MM, Cuthbert BN. International Affective Picture System (IAPS): instruction manual and affective ratings. Technical Report A-4. Gainesville: University of Florida; 1999.

10. Lang PJ, Bradley MM, Cuthbert BN. International Affective Picture System (IAPS): affective ratings of pictures and instruction manual. Technical Report A-8. Gainesville: University of Florida; 2008.

11. Bradley MM, Lang PJ. International Affective Digitized Sounds (IADS): stimuli, instruction manual and affective ratings. Technical Report B-2. Gainesville: University of Florida; 1999.

12. Bradley MM, Lang PJ. Affective Norms for English Text (ANET): affective ratings of text and instruction manual. Technical Report D-1. Gainesville: University of Florida; 2007.

13. Bradley MM, Lang PJ. Affective Norms for English Words (ANEW): instruction manual and affective ratings. Technical Report C-1. Gainesville: University of Florida; 1999.

14. Lang PJ. Emotion and motivation: toward consensus definitions and a common research purpose. Emot Rev. 2010;2:229-33.

15. Osgood C, Suci GL, Tannenbaum P. The measurement of meaning. Urbana: University of Illinois; 1957.

16. Russell JA. A circumplex model of affect. J Pers Soc Psychol. 1980;39:1161-78.

17. Bradley MM, Lang PJ. Affective reactions to acoustic stimuli. Psychophysiology. 2000;37:204-15.

18. Flaisch T, Junghofer M, Bradley MM, Schupp HT, Lang PJ. Rapid picture processing: affective primes and targets. Psychophysiology. 2008;45:1-10.

19. Flor H, Birbaumer N, Hermann C, Ziegler S, Patrick CJ. Aversive Pavlovian conditioning in psychopaths: peripheral and central correlates. Psychophysiology. 2002;39:505-18.

20. McTeague LM, Lang PJ, Laplante M-C, Cuthbert BN, Shumen $\mathrm{JR}$, Bradley MM. Aversive imagery in posttraumatic stress disorder: trauma recurrence, comorbidity, and physiological reactivity. Biol Psychiatry. 2010;67:346-56.

21. Ressler KJ, Rothbaum BO, Tannenbaum L, Anderson P, Graap $K$, Zimand $E$, et al. Cognitive enhancers as adjuncts to psychotherapy: use of $D$-cycloserine in phobic individuals to facilitate extinction of fear. Arch Gen Psychiatry. 2004;61:1136-44.

22. Gross JJ. The emerging field of emotion regulation: an integrative review. Rev Gen Psychol. 1998;2:271-99.

23. Ochsner KN, Gross JJ. The cognitive control of emotion. Trends Cogn Sci. 2005;9:242-9.

24. Cicchetti D, Ackerman BP, Izard CE. Emotions and emotion regulation in developmental psychopathology. Dev Psychopathol. 1995; 7:1-10.

25. Mennin DS, Holoway RM, Fresco DM, Moore MT, Heimberg RG. Delineating components of emotion and its dysregulation in anxiety and mood psychopathology. Behav Ther. 2007; 38:284-302.

26. Watson D. Rethinking the mood and anxiety disorders: a quantitative hierarchical model for DSM-V. J Abnorm Psychol. 2005; 114:522-36.

27. Lasaitis C, Ribeiro RL, Bueno OFA. Brazilian norms for the International Affective Picture System (IAPS): comparison of the affective ratings for new stimuli between Brazilian and North American subjects. J Bras Psiquiatr. 2008;57:270-5.

28. Pôrto WG, Bertolucci PHF, Bueno OFA. The paradox of age: an analysis of responses by aging Brazilians to the International Affective Picture System (IAPS). Rev Bras Psiquiatr. 2011;33:10-5.

29. Ribeiro RL, Pompéia S, Bueno OFA. Normas brasileiras para o International Affective Picture System (IAPS): comunicação breve. Rev Psiquiatr Rio Gd Sul. 2004;26:190-4.

30. Kensinger EA. Age differences in memory for arousing and nonarousing emotional words. J Gerontol. 2008;63:13-8. 
31. Ashley V, Swick D. Consequences of emotional stimuli: age differences on pure and mixed blocks of the emotional Stroop. Behav Brain Funct. 2009;5:14.

32. van Hooff JC, Dietz KC, Sharma D, Bowman H. Neural correlates of intrusion of emotion words in a modified Stroop task. Int J Psychophysiol. 2008;67:23-34.

33. Bonin P, Méot A, Aubert L, Malardier N, Niedenthal PM, Capelle-Toczek M-C. Normes de concrétude, de valeur dimagerie, de fréquence subjective et de valence émotionnelle pour 866 mots. Annee Psychol. 2003;103:655-94.

34. Syssau A, Font N. Evaluations des caractéristiques émotionnelles d'un corpus de 604 mots. Bull Psychol. 2005;58:361-7.

35. Võ ML-H, Jacobs AM, Conrad M. Cross-validating the Berlin Affective Word List. Behav Res Methods. 2006;38:606-9.

36. Võ ML-H, Conrad M, Kuchinke L, Urton K, Hofmann MJ, Jacobs AM. The Berlin Affective Word List Reloaded (BAWL-R). Behav Res Methods. 2009; 41:534-8.

37. Redondo J, Fraga I, Padrón I, Comesaña M. The Spanish adaptation of ANEW (Affective Norms for English Words). Behav Res Methods. 2007;39:600-5.

38. Eilola TM, Havelka J. Affective norms for 210 British English and Finnish nouns. Behav Res Methods. 2010;42:134-40.

39. Kensinger EA, Brierley B, Medford N, Growdon JH, Corkin S. Effects of normal aging and Alzheimer's disease on emotional memory. Emotion. 2002;2:118-34.

40. Danion JM, Kazes M, Huron C, Karchouni N. Do patients with schizophrenia consciously recollect emotional events better than neutral events? Am J Psychiatry. 2003;160:1879-81.

41. Buchanan TW, Etzel JA, Adolphs R, Tranel D. The influence of autonomic arousal and semantic relatedness on memory for emotional words. Int J Psychophysiology. 2006;61:26-33.
42. Santos RF, Silveira RAT, Gomes CFA, Stein LM. Normas de emocionalidade para a versão brasileira do paradigma Deese-Roediger-McDermott (DRM). Psicol Teor Pesq. 2009;25:387-94.

43. Stein LM, Gomes CFA. Normas brasileiras para listas de palavras associadas: associação semântica, concretude, frequência e emocionalidade. Psicol Teor Pesq. 2009;25:537-46.

44. Greenwald MK, Cook EW, Lang PJ. Affective judgment and psychophysiological response: dimensional covariation in the evaluation of pictorial stimuli. J Psychophysiol. 1989;3:51-64.

45. Lang PJ, Greenwald MK, Bradley MM, Harem AO. Looking at pictures: affective, visceral, and behavioral reactions. Psychophysiology. 1993;30:261-73.

46. Lewis PA, Critchley HD, Rotshtein P, Dolan RJ. Neural correlates of processing valence and arousal in affective words. Cereb Cortex. 2007; 17:742-8.

47. Holland AC, Kensinger EA. Emotion and autobiographical memory. Phys Life Rev. 2010;7:88-131.

48. LaBar KS, Cabeza R. Cognitive neuroscience of emotional memory. Nat Rev Neurosci. 2006;7:54-64.

49. Cahill L, Haier RJ, Fallon J, Alkire MT, Tang C, Keator D, et al. Amygdala activity at encoding correlated with long-term, free recall of emotional information. Proc Natl Acad Sci U S A. $1996 ; 93: 8016-21$.

\section{Correspondência}

Christian Haag Kristensen

Av. Ipiranga, 6681, prédio 11 , sala 933, Partenon

90619-900 - Porto Alegre, RS - Brasil

Tel.: (51) 33203500

E-mail: christian.kristensen@pucrs.br 
Anexo 1 - Versão brasileira do Affective Norms for English Words (ANEW-Br)

\begin{tabular}{|c|c|c|c|c|c|c|c|c|c|c|c|c|c|c|}
\hline \multirow[b]{2}{*}{ Palavra } & \multicolumn{2}{|c|}{ Valência } & \multicolumn{2}{|c|}{ Alerta } & \multirow[b]{2}{*}{ Palavra } & \multicolumn{2}{|c|}{ Valência } & Ale & rta & & Valê & ncia & Ale & rta \\
\hline & Média & DP & Média & DP & & Média & DP & Média & DP & Palavra & Média & DP & Média & DP \\
\hline ABALADO & 2,58 & 1,81 & 5,11 & 2,82 & ARTE & 8,08 & 1,50 & 3,68 & 3,21 & CACHOEIRA & 8,14 & 1,65 & 3,84 & 3,35 \\
\hline ABANDONADO & 1,85 & 1,70 & 5,48 & 2,49 & ÁRVORE & 7,98 & 1,46 & 2,27 & 2,19 & CACHORRO & 7,98 & 1,46 & 3,98 & 2,80 \\
\hline ABELHAS & 4,58 & 2,45 & 4,38 & 2,83 & ÁSPERO & 3,38 & 2,34 & 5,49 & 2,53 & CADEIA & 2,02 & 1,97 & 5,70 & 3,20 \\
\hline ABENÇOADO & 8,03 & 1,84 & 3,19 & 3,03 & ASSALTANTE & 2,03 & 2,25 & 7,24 & 2,70 & CADEIRA & 6,30 & 1,81 & 2,76 & 1,72 \\
\hline ABORTO & 1,67 & 1,37 & 6,42 & 2,93 & ASSALTO & 1,47 & 1,10 & 7,67 & 2,25 & CADERNO & 5,76 & 1,85 & 4,02 & 2,15 \\
\hline ABRAÇAR & 8,63 & 1,20 & 4,30 & 3,34 & ASSAR & 6,24 & 2,73 & 3,92 & 2,70 & CAIXÃO & 1,92 & 1,85 & 6,08 & 3,13 \\
\hline ABRASADOR & 5,43 & 1,91 & 3,53 & 2,49 & ASSENTO & 5,87 & 2,07 & 3,05 & 2,20 & CAMA & 8,08 & 1,36 & 3,28 & 2,93 \\
\hline ABRIGADO & 7,61 & 1,98 & 3,21 & 2,41 & ASSOVIO & 6,15 & 2,15 & 4,48 & 2,62 & CAMINHÃO & 4,96 & 1,76 & 4,27 & 2,25 \\
\hline ABSURDO & 2,79 & 2,12 & 5,81 & 2,74 & ASSUSTADO & 2,89 & 2,17 & 6,00 & 2,54 & CAMPEÃO & 8,43 & 1,21 & 5,03 & 3,34 \\
\hline ABUNDÂNCIA & 6,94 & 2,05 & 4,19 & 2,48 & ASTRONAUTA & 5,81 & 1,66 & 3,84 & 2,45 & CAMPO & 7,61 & 1,78 & 3,09 & 2,79 \\
\hline ABUSO & 2,76 & 2,65 & 6,50 & 2,65 & ATADURA & 2,87 & 2,26 & 4,50 & 2,89 & CANÇÃO & 8,35 & 1,53 & 3,70 & 3,31 \\
\hline ACALMAR & 7,23 & 1,81 & 2,68 & 2,05 & ATERRORIZADO & 1,79 & 1,46 & 7,32 & 2,53 & CÂNCER & 1,49 & 1,19 & 6,98 & 2,84 \\
\hline ACANHADO & 3,89 & 2,01 & 5,03 & 2,75 & ATIVAR & 7,19 & 1,82 & 4,19 & 2,60 & CANHÃO & 2,38 & 1,99 & 5,78 & 2,62 \\
\hline ACASO & 6,17 & 2,10 & 4,28 & 2,48 & ATLETISMO & 7,31 & 2,13 & 4,27 & 2,50 & CANSADO & 2,39 & 1,88 & 5,39 & 3,26 \\
\hline ACEITAÇÃO & 7,21 & 2,16 & 4,63 & 2,94 & ATRAÇÃO & 8,22 & 1,49 & 4,84 & 3,43 & CAOS & 2,05 & 2,00 & 6,51 & 2,79 \\
\hline ACONCHEGANTE & 8,29 & 1,69 & 3,76 & 2,94 & AUTONOMIA & 7,71 & 1,92 & 4,29 & 3,10 & CARCAÇA & 3,75 & 2,23 & 4,29 & 2,72 \\
\hline ACONCHEGO & 8,40 & 1,01 & 2,68 & 2,67 & AVALANCHE & 2,32 & 2,10 & 6,02 & 2,90 & CÁRCERE & 1,89 & 1,55 & 5,95 & 2,84 \\
\hline ACORDO & 6,89 & 2,01 & 3,37 & 2,25 & AVENIDA & 5,84 & 1,84 & 3,76 & 2,40 & CARÍCIA & 8,78 & 0,58 & 4,42 & 3,51 \\
\hline AÇÚCAR & 7,04 & 1,71 & 3,91 & 2,58 & AVENTURA & 8,06 & 1,53 & 6,33 & 2,97 & CÁRIE & 2,27 & 1,92 & 5,71 & 2,91 \\
\hline ADAGA & 4,32 & 2,30 & 4,27 & 2,46 & AVÔ & 7,18 & 2,80 & 4,05 & 2,90 & CARINHOSO & 8,73 & 0,80 & 4,42 & 3,52 \\
\hline ADMIRADO & 7,67 & 1,53 & 4,49 & 2,82 & AZEDO & 2,87 & 2,04 & 4,42 & 2,13 & CAROÇO & 3,81 & 1,83 & 4,49 & 2,77 \\
\hline ADORÁVEL & 8,24 & 1,57 & 4,00 & 3,26 & AZUL & 7,42 & 2,32 & 3,37 & 2,78 & CARRO & 7,95 & 1,78 & 4,36 & 3,19 \\
\hline ADULTO & 6,49 & 1,99 & 3,75 & 2,46 & BACIA & 4,68 & 1,84 & 3,59 & 2,53 & CARTA & 7,02 & 2,05 & 4,20 & 2,37 \\
\hline AFEIÇÃO & 7,82 & 1,90 & 3,84 & 2,89 & BAGUNÇADO & 3,21 & 2,28 & 5,92 & 2,61 & CASA & 8,14 & 1,58 & 3,35 & 2,99 \\
\hline AFINAR & 5,80 & 1,54 & 3,65 & 2,09 & BALA & 6,98 & 1,99 & 3,44 & 2,45 & CASAL & 7,84 & 1,91 & 4,09 & 3,05 \\
\hline AFOGAR & 2,53 & 2,26 & 6,24 & 2,61 & BANDEIRA & 6,32 & 1,65 & 3,00 & 2,25 & CASAMENTO & 7,59 & 2,03 & 4,57 & 3,27 \\
\hline AGILIDADE & 7,50 & 1,58 & 5,33 & 2,84 & BANHEIRA & 8,00 & 1,37 & 3,00 & 2,69 & CASSINO & 5,21 & 2,71 & 4,98 & 2,71 \\
\hline AGONIA & 2,05 & 1,97 & 6,61 & 2,70 & BANHEIRO & 6,82 & 1,72 & 3,00 & 2,51 & CAVALO & 6,89 & 1,93 & 3,24 & 2,68 \\
\hline AGRADÁVEL & 8,15 & 1,25 & 3,18 & 2,49 & BANHO & 8,29 & 1,50 & 2,61 & 2,74 & CAVEIRA & 2,61 & 1,77 & 5,07 & 2,75 \\
\hline AGRADECIDO & 8,42 & 1,52 & 3,32 & 2,98 & BANQUETA & 5,84 & 1,98 & 2,61 & 1,76 & CÉDULA & 7,43 & 2,10 & 4,76 & 2,86 \\
\hline AGRESSIVO & 1,89 & 1,66 & 6,67 & 2,99 & BARATA & 2,83 & 1,89 & 5,23 & 3,17 & CEFALÉIA & 2,92 & 1,89 & 5,47 & 2,67 \\
\hline ÁGUA & 8,35 & 1,40 & 3,00 & 2,90 & BARRA & 4,74 & 1,91 & 4,37 & 2,38 & CEGO & 2,35 & 1,87 & 4,68 & 2,65 \\
\hline AGULHA & 4,04 & 1,98 & 4,93 & 2,54 & BARRIL & 5,85 & 2,11 & 4,28 & 2,58 & CÉLULA & 5,06 & 2,06 & 3,02 & 2,20 \\
\hline ALERGIA & 4,02 & 3,31 & 5,20 & 3,00 & BECO & 3,02 & 2,06 & 5,80 & 2,90 & CÉU & 8,10 & 1,21 & 2,76 & 2,30 \\
\hline ALERTA & 6,08 & 2,42 & 5,08 & 2,94 & BEIJO & 8,76 & 0,79 & 5,24 & 3,74 & CHACINA & 1,43 & 1,48 & 6,86 & 2,78 \\
\hline ALERTO & 5,67 & 2,06 & 4,95 & 2,64 & BELEZA & 8,09 & 1,25 & 3,91 & 2,84 & CHALEIRA & 5,19 & 1,70 & 3,02 & 2,16 \\
\hline ALIMENTO & 8,26 & 1,27 & 3,61 & 3,07 & BELISCAR & 3,71 & 2,37 & 4,82 & 2,66 & CHAMUSCAR & 4,46 & 2,10 & 4,24 & 2,37 \\
\hline ALVORECER & 6,24 & 2,38 & 3,09 & 2,22 & BELO & 7,73 & 1,77 & 3,29 & 2,53 & CHANTAGEM & 1,61 & 1,44 & 6,84 & 2,77 \\
\hline AMABILIDADE & 7,68 & 2,04 & 3,50 & 2,96 & BENZER & 6,50 & 2,06 & 2,76 & 2,68 & CHAPÉU & 6,16 & 1,85 & 2,54 & 1,74 \\
\hline AMADO & 8,31 & 1,39 & 3,49 & 3,00 & BERÇÁRIO & 7,60 & 1,70 & 3,30 & 2,33 & CHARME & 7,63 & 1,77 & 5,35 & 2,75 \\
\hline AMARELO & 6,76 & 2,29 & 3,11 & 2,38 & BERRAR & 3,63 & 2,36 & 6,21 & 2,73 & CHATEAÇÃO & 2,30 & 2,28 & 6,00 & 2,70 \\
\hline AMÁVEL & 8,09 & 1,60 & 3,40 & 2,88 & BESTA & 2,45 & 1,93 & 5,30 & 2,76 & CHAVE & 5,46 & 1,79 & 3,36 & 2,40 \\
\hline AMBIÇÃO & 5,00 & 3,08 & 5,92 & 3,00 & BISPO & 5,68 & 2,51 & 2,71 & 2,40 & CHOCALHO & 5,73 & 1,94 & 3,12 & 2,29 \\
\hline AMBULÂNCIA & 2,47 & 2,28 & 6,30 & 3,00 & BLASFÊMIA & 2,63 & 1,78 & 5,71 & 2,60 & CHOCOLATE & 8,08 & 1,40 & 4,88 & 3,03 \\
\hline AMEAÇA & 1,87 & 1,68 & 6,71 & 2,73 & BOBAGEM & 4,84 & 2,48 & 4,76 & 2,78 & CHUTE & 4,40 & 2,40 & 4,27 & 2,75 \\
\hline AMIGÁVEL & 8,25 & 1,34 & 3,36 & 2,93 & BOLINHO & 7,50 & 1,54 & 3,40 & 2,47 & CHUVA & 5,54 & 2,51 & 4,47 & 2,73 \\
\hline AMIGO & 8,74 & 0,79 & 3,68 & 3,24 & BOLO & 7,37 & 2,01 & 3,26 & 2,64 & CICATRIZ & 3,23 & 1,99 & 3,98 & 2,60 \\
\hline AMOR & 8,75 & 0,89 & 4,39 & 3,57 & $\mathrm{BOM}$ & 8,19 & 1,53 & 3,36 & 2,65 & CICLONE & 2,67 & 1,99 & 6,04 & 2,77 \\
\hline ANGUSTIADO & 2,22 & 1,90 & 6,65 & 2,32 & BOMBA & 1,53 & 1,39 & 6,87 & 2,56 & CIDADE & 6,41 & 2,12 & 4,71 & 2,70 \\
\hline ANIMAÇÃO & 8,33 & 1,43 & 4,64 & 3,03 & BONECA & 5,57 & 2,19 & 3,05 & 2,24 & CINEMA & 8,25 & 1,40 & 4,75 & 3,30 \\
\hline ANIVERSÁRIO & 8,39 & 1,22 & 4,71 & 3,34 & BONITO & 8,42 & 1,41 & 3,45 & 2,94 & CIRCO & 6,77 & 2,08 & 3,10 & 2,49 \\
\hline ANJO & 8,06 & 1,59 & 2,45 & 2,16 & BORBOLETA & 7,38 & 1,82 & 2,56 & 1,88 & CÍRCULO & 5,29 & 1,49 & 3,71 & 2,31 \\
\hline ANSEIO & 5,11 & 2,48 & 5,18 & 2,50 & BOXEADOR & 4,13 & 2,50 & 5,34 & 2,50 & CIRURGIA & 2,85 & 2,34 & 5,81 & 2,80 \\
\hline ANSIOSO & 3,50 & 2,44 & 7,05 & 2,44 & BRABEZA & 2,83 & 2,15 & 6,02 & 2,71 & CIÚME & 2,86 & 1,84 & 6,05 & 2,45 \\
\hline APARELHO & 6,00 & 2,29 & 3,13 & 2,58 & BRABO & 2,26 & 1,83 & 6,05 & 2,70 & COBERTURA & 7,33 & 1,74 & 3,57 & 3,08 \\
\hline APÁTICO & 3,21 & 2,02 & 4,93 & 2,69 & BRAÇO & 6,35 & 2,12 & 3,64 & 2,12 & COBRA & 2,68 & 1,86 & 6,16 & 2,73 \\
\hline APLAUSO & 8,21 & 1,17 & 4,95 & 3,25 & BRANCO & 7,18 & 2,08 & 2,61 & 2,47 & COELHINHO & 7,31 & 1,68 & 2,83 & 2,50 \\
\hline APRENDER & 7,86 & 1,81 & 4,78 & 3,03 & BRAVO & 2,56 & 2,20 & 6,31 & 2,87 & COELHO & 6,96 & 1,70 & 3,41 & 2,50 \\
\hline AR & 8,39 & 1,24 & 2,57 & 2,72 & BRILHANTE & 7,45 & 1,81 & 3,21 & 2,43 & COFRE & 6,13 & 1,83 & 3,63 & 2,84 \\
\hline ARANHA & 3,49 & 2,26 & 5,62 & 2,85 & BRINQUEDO & 7,54 & 1,77 & 3,56 & 2,45 & COGUMELO & 5,35 & 1,64 & 3,35 & 2,36 \\
\hline ARMA & 2,24 & 2,50 & 6,97 & 2,50 & BRISA & 7,39 & 2,11 & 2,76 & 2,80 & COLETE & 4,63 & 1,92 & 3,00 & 2,33 \\
\hline ARMAMENTO & 2,17 & 1,65 & 6,45 & 2,97 & BRUTAL & 1,98 & 1,84 & 6,60 & 2,77 & COLISÃO & 2,40 & 1,75 & 6,16 & 2,66 \\
\hline ARMÁRIO & 6,11 & 1,72 & 3,21 & 2,20 & BUQUÊ & 7,42 & 2,41 & 3,74 & 3,19 & COLUNA & 4,65 & 2,10 & 3,92 & 3,03 \\
\hline ARREPENDIDO & 3,46 & 2,46 & 5,20 & 2,64 & BURRO & 2,96 & 1,90 & 4,70 & 2,73 & COMÉDIA & 8,39 & 1,25 & 4,40 & 3,29 \\
\hline ARROGANTE & 2,05 & 1,90 & 6,32 & 2,77 & CABANA & 7,21 & 1,74 & 3,21 & 2,68 & COMER & 7,42 & 2,22 & 4,58 & 2,94 \\
\hline ARRUMADO & 7,78 & 1,82 & 3,24 & 2,39 & CABELO & 6,67 & 2,41 & 3,78 & 2,64 & COMPLACENTE & 4,71 & 1,50 & 3,95 & 2,26 \\
\hline
\end{tabular}


Anexo 1 - Versão brasileira do

Affective Norms for English Words (ANEW-Br) (cont.)

\begin{tabular}{|c|c|c|c|c|c|c|c|c|c|c|c|c|c|c|}
\hline \multirow[b]{2}{*}{ Palavra } & \multicolumn{2}{|c|}{ Valência } & \multicolumn{2}{|c|}{ Alerta } & \multirow[b]{2}{*}{ Palavra } & \multicolumn{2}{|c|}{ Valência } & Ale & & & Valê & cia & Ale & rta \\
\hline & Média & DP & Média & DP & & Média & DP & Média & DP & Palavra & Média & DP & Média & DP \\
\hline COMPROMETIDO & 6,33 & 2,53 & 4,65 & 3,08 & DESAJEITADO & 3,67 & 1,95 & 3,65 & 2,40 & ESCORPIÃO & 3,53 & 2,11 & 4,61 & 2,91 \\
\hline COMPUTADOR & 6,62 & 1,98 & 4,66 & 2,62 & DESAMPARADO & 2,48 & 1,65 & 5,00 & 2,62 & ESCRAVO & 2,36 & 2,21 & 5,82 & 2,93 \\
\hline CONFIANÇA & 7,97 & 2,08 & 4,67 & 3,31 & DESASTRE & 1,84 & 1,56 & 5,97 & 2,93 & ESCRITÓRIO & 5,70 & 2,18 & 4,41 & 2,39 \\
\hline CONFIANTE & 7,42 & 1,99 & 4,64 & 3,09 & DESAVENÇA & 1,98 & 1,42 & 5,44 & 2,83 & ESCURO & 4,49 & 2,26 & 3,67 & 2,86 \\
\hline CONFORTO & 8,03 & 1,53 & 4,17 & 3,12 & DESCONFORTO & 2,31 & 1,76 & 5,60 & 2,86 & ESFERA & 5,43 & 1,65 & 3,53 & 2,43 \\
\hline CONFUSO & 2,54 & 1,61 & 5,83 & 3,00 & DESCULPA & 5,90 & 2,67 & 4,37 & 2,83 & ESFOMEADO & 3,61 & 2,66 & 5,22 & 2,89 \\
\hline CÔNJUGE & 6,02 & 2,52 & 4,23 & 3,18 & DESEJO & 7,78 & 1,72 & 5,06 & 2,90 & ESNOBE & 1,96 & 1,41 & 5,22 & 3,03 \\
\hline CONSOLADO & 5,36 & 2,11 & 3,55 & 2,44 & DESERTOR & 3,72 & 1,59 & 4,58 & 2,19 & ESPAÇO & 6,88 & 2,13 & 4,39 & 2,79 \\
\hline CONSTRANGIDO & 2,96 & 1,41 & 5,15 & 2,73 & DESESPERADOR & 1,92 & 1,58 & 6,12 & 3,11 & ESPANTADO & 4,47 & 1,93 & 4,80 & 2,87 \\
\hline CONTENTAMENTO & 6,72 & 2,33 & 4,18 & 2,84 & DESINTERESSADO & 3,46 & 1,90 & 5,16 & 2,65 & ESPERANÇA & 8,29 & 1,37 & 5,04 & 3,11 \\
\hline CONTEÚDO & 7,04 & 1,87 & 3,81 & 2,60 & DESLEAL & 1,63 & 1,30 & 5,90 & 3,17 & ESPERANÇOSO & 7,55 & 1,87 & 4,08 & 2,76 \\
\hline CONTEXTO & 5,36 & 1,56 & 3,99 & 2,32 & DESLIGADO & 3,31 & 1,86 & 5,13 & 2,64 & ESPINGARDA & 2,82 & 2,28 & 5,66 & 2,85 \\
\hline CONTINÊNCIA & 4,40 & 1,91 & 3,81 & 2,69 & DESPEJAR & 4,08 & 2,04 & 3,92 & 2,81 & ESPINHO & 2,98 & 1,70 & 4,45 & 2,89 \\
\hline CONTROLE & 5,75 & 2,28 & 5,04 & 2,65 & DESPERDÍCIO & 3,45 & 1,89 & 4,99 & 2,50 & ESPÍRITO & 6,87 & 2,04 & 4,27 & 2,70 \\
\hline COR & 7,65 & 1,63 & 3,48 & 2,94 & DESPREOCUPADO & 5,33 & 2,70 & 3,35 & 2,45 & ESPOSA & 5,80 & 2,34 & 3,71 & 2,72 \\
\hline CORAGEM & 7,96 & 1,65 & 5,19 & 3,01 & DESPREZO & 1,71 & 1,32 & 4,96 & 3,18 & ESQUINA & 4,67 & 1,45 & 3,27 & 2,48 \\
\hline CORDA & 4,53 & 1,72 & 3,96 & 2,36 & DESTACADO & 6,82 & 1,81 & 4,53 & 2,57 & ESTAGNADO & 3,54 & 1,97 & 4,86 & 2,31 \\
\hline CORDEIRO & 5,56 & 2,05 & 2,58 & 2,29 & DESTROÇAR & 2,53 & 1,79 & 4,39 & 3,00 & ESTÁTUA & 4,88 & 2,06 & 2,53 & 2,22 \\
\hline COROA & 5,58 & 1,94 & 3,76 & 2,37 & DESTRUIÇÃO & 2,13 & 1,93 & 5,88 & 2,81 & ESTERCO & 3,26 & 1,93 & 3,76 & 2,60 \\
\hline CORPO & 6,94 & 2,34 & 4,67 & 2,79 & DESTRUIR & 2,00 & 1,68 & 5,35 & 3,17 & ESTÔMAGO & 5,14 & 2,15 & 3,71 & 2,45 \\
\hline CORREDOR & 5,30 & 1,76 & 4,27 & 2,54 & DETALHE & 6,36 & 1,80 & 4,40 & 2,67 & ESTRANGEIRO & 6,00 & 1,91 & 4,22 & 2,48 \\
\hline CORRUPTO & 1,63 & 1,18 & 6,04 & 3,11 & DETESTAR & 2,29 & 1,71 & 4,39 & 3,08 & ESTRANHO & 4,58 & 1,73 & 5,16 & 1,93 \\
\hline CORTE & 3,30 & 1,81 & 5,09 & 2,69 & DEUS & 8,11 & 1,54 & 3,80 & 3,15 & ESTRELA & 7,75 & 2,01 & 3,57 & 3,07 \\
\hline CORTESIA & 8,02 & 1,42 & 3,60 & 2,96 & DEVOTADO & 5,80 & 2,01 & 2,82 & 2,37 & ESTRESSE & 2,22 & 1,77 & 6,51 & 2,77 \\
\hline CORTIÇA & 5,00 & 1,32 & 3,68 & 2,31 & DIABO & 2,67 & 2,20 & 4,63 & 2,86 & ESTUPENDO & 3,88 & 3,47 & 6,67 & 3,03 \\
\hline CORTINAS & 5,88 & 1,84 & 2,42 & 2,04 & DIAMANTE & 7,22 & 2,09 & 4,10 & 2,88 & ESTÚPIDO & 2,21 & 1,98 & 6,18 & 2,71 \\
\hline CORUJA & 5,68 & 1,86 & 3,57 & 2,39 & DIGNO & 7,69 & 1,67 & 4,01 & 2,75 & ESTUPRO & 4,94 & 3,29 & 5,10 & 2,90 \\
\hline COSTA & 5,54 & 1,92 & 3,50 & 2,58 & DINHEIRO & 7,20 & 2,52 & 5,39 & 3,03 & EVENTO & 7,03 & 2,40 & 4,51 & 2,93 \\
\hline COSTUME & 5,42 & 1,82 & 3,87 & 2,45 & DIPLOMA & 8,33 & 1,39 & 6,03 & 3,05 & EXAME & 4,22 & 2,13 & 3,93 & 11,52 \\
\hline COTOVELO & 4,88 & 1,72 & 2,40 & 2,02 & DIVERSÃO & 8,31 & 1,76 & 5,16 & 3,20 & EXCELÊNCIA & 6,24 & 2,13 & 4,35 & 2,41 \\
\hline COVARDE & 2,51 & 1,77 & 5,45 & 2,66 & DIVERTIDO & 8,57 & 1,26 & 5,12 & 3,42 & EXCITAÇÃO & 7,73 & 1,75 & 6,26 & 3,01 \\
\hline COZINHEIRO & 6,79 & 2,00 & 3,40 & 2,80 & DIVERTIMENTO & 8,27 & 1,56 & 4,88 & 3,24 & EXCURSÃO & 7,82 & 2,04 & 4,85 & 3,15 \\
\hline CREPÚSCULO & 5,34 & 2,06 & 4,28 & 2,54 & DIVÓRCIO & 2,97 & 2,06 & 5,29 & 2,96 & EXECUÇÃO & 3,92 & 2,92 & 4,79 & 2,75 \\
\hline CRISE & 2,31 & 1,73 & 5,97 & 2,73 & DÓLAR & 5,45 & 2,44 & 5,09 & 2,73 & EXULTANTE & 5,97 & 2,10 & 4,29 & 2,71 \\
\hline CRU & 3,58 & 2,18 & 3,10 & 2,69 & DOMINADOR & 3,96 & 2,15 & 4,71 & 2,80 & FACA & 3,90 & 1,96 & 5,21 & 2,45 \\
\hline CRUCIFICAR & 2,36 & 1,62 & 5,32 & 2,97 & DOR & 1,91 & 1,65 & 5,97 & 2,87 & FÁCIL & 7,31 & 2,16 & 3,49 & 2,91 \\
\hline CRUEL & 1,67 & 1,39 & 5,58 & 3,10 & DOUTOR & 4,84 & 2,22 & 3,86 & 2,68 & FAIXA & 4,67 & 1,14 & 4,24 & 1,64 \\
\hline CULINÁRIA & 7,03 & 2,23 & 4,51 & 3,05 & DURO & 4,41 & 2,13 & 4,60 & 2,64 & FALCÃO & 5,82 & 2,04 & 3,97 & 2,52 \\
\hline CULPADO & 2,27 & 1,57 & 5,19 & 2,84 & EDIFÍCIO & 5,76 & 1,88 & 2,49 & 1,80 & FALHA & 2,03 & 1,46 & 6,93 & 2,18 \\
\hline CUPIM & 2,99 & 1,85 & 4,56 & 3,03 & EDUCAÇÃO & 8,04 & 1,75 & 4,92 & 3,07 & FALIDO & 2,00 & 1,73 & 5,40 & 3,06 \\
\hline CURAR & 7,83 & 1,74 & 4,42 & 3,09 & EGOÍSTA & 2,04 & 1,78 & 5,33 & 2,89 & FALSO & 5,45 & 3,59 & 5,03 & 3,27 \\
\hline CURIOSO & 6,77 & 1,82 & 5,48 & 2,57 & ELEGANTE & 7,38 & 1,76 & 4,62 & 2,68 & FAMA & 6,73 & 2,04 & 5,38 & 2,76 \\
\hline DÁDIVA & 7,73 & 1,75 & 3,31 & 2,81 & ELEVADOR & 5,37 & 1,98 & 3,24 & 2,39 & FAMÍLIA & 7,07 & 2,22 & 4,09 & 3,05 \\
\hline DANÇARINO & 6,70 & 2,20 & 4,33 & 3,09 & EMPREGO & 7,31 & 2,18 & 5,97 & 2,88 & FAMINTO & 2,94 & 2,54 & 5,70 & 2,89 \\
\hline DANO & 2,32 & 1,78 & 4,72 & 2,69 & ENCARDIDO & 3,08 & 1,59 & 4,02 & 2,53 & FAMOSO & 5,76 & 2,08 & 4,24 & 2,23 \\
\hline DÉBIL & 3,13 & 1,83 & 3,86 & 2,55 & ENCHARPE & 5,59 & 1,80 & 3,64 & 2,34 & FANTASIA & 7,06 & 2,08 & 5,18 & 2,90 \\
\hline DÉBITO & 2,47 & 1,82 & 5,30 & 2,84 & ENCONTRO & 7,67 & 2,10 & 5,67 & 2,86 & FAROL & 6,75 & 1,99 & 5,00 & 2,70 \\
\hline DECEPCIONAR & 1,76 & 1,28 & 6,09 & 2,70 & ENFERMEIRA & 5,33 & 2,20 & 4,39 & 2,47 & FAROLETE & 5,20 & 1,81 & 3,40 & 2,26 \\
\hline DECOMPOR & 3,94 & 2,06 & 2,92 & 2,24 & ENFERRUJADO & 3,41 & 1,68 & 3,25 & 2,18 & FASCINAR & 5,37 & 2,65 & 4,39 & 2,51 \\
\hline DECORAR & 6,22 & 2,78 & 4,72 & 2,90 & ENFURECIDO & 2,67 & 2,09 & 6,89 & 2,17 & FASE & 5,44 & 2,24 & 4,33 & 2,65 \\
\hline DEDO & 5,75 & 1,87 & 2,56 & 2,31 & ENGANAÇÃO & 1,76 & 1,56 & 6,31 & 2,91 & FATIGADO & 5,10 & 2,97 & 4,72 & 3,06 \\
\hline DEFEITO & 3,15 & 1,99 & 5,17 & 2,54 & ENJOATIVO & 2,63 & 1,50 & 4,72 & 2,45 & FAVELA & 1,64 & 1,25 & 5,63 & 2,96 \\
\hline DEFICIENTE & 3,13 & 1,91 & 4,13 & 2,92 & ENLAMEADO & 3,57 & 1,83 & 3,65 & 2,54 & FAVOR & 7,62 & 1,78 & 3,75 & 2,71 \\
\hline DEFORMADO & 2,43 & 1,78 & 4,95 & 2,89 & ENTEDIADO & 2,44 & 1,92 & 5,19 & 2,83 & FAVORITO & 7,27 & 2,07 & 5,12 & 2,84 \\
\hline DELEITE & 5,53 & 2,14 & 2,82 & 2,37 & ENTERRO & 1,65 & 1,32 & 5,31 & 3,31 & FAZENDA & 4,32 & 3,12 & 4,43 & 3,08 \\
\hline DELICADO & 6,30 & 2,11 & 3,72 & 2,57 & ENTULHO & 3,61 & 2,12 & 3,54 & 6,56 & FEBRE & 2,20 & 1,65 & 4,90 & 2,91 \\
\hline DEMÔNIO & 2,10 & 1,67 & 4,49 & 3,25 & ENTUSIASMO & 8,02 & 1,83 & 4,78 & 3,11 & FEDOR & 5,58 & 3,61 & 5,43 & 3,43 \\
\hline DEMORADO & 2,94 & 2,14 & 6,00 & 2,81 & ENVERGONHADO & 3,29 & 1,95 & 5,33 & 2,42 & FEITO & 3,17 & 2,25 & 4,15 & 2,64 \\
\hline DENTISTA & 5,18 & 2,51 & 4,02 & 2,65 & ERÓTICO & 7,31 & 2,03 & 4,80 & 3,21 & FELIZ & 8,69 & 1,00 & 4,13 & 3,44 \\
\hline DEPRESSÃO & 1,96 & 1,55 & 5,69 & 3,05 & ERRO & 2,43 & 1,91 & 6,40 & 2,57 & FENO & 4,65 & 1,62 & 3,24 & 2,29 \\
\hline DEPRIMENTE & 1,82 & 1,29 & 4,88 & 3,11 & ERUDITO & 5,47 & 1,97 & 2,86 & 2,31 & FERIADO & 4,99 & 3,49 & 4,55 & 3,23 \\
\hline DEPRIMIDO & 2,20 & 1,58 & 5,12 & 2,73 & ESBANJAMENTO & 3,47 & 2,29 & 5,25 & 2,64 & FÉRIAS & 8,62 & 1,50 & 5,87 & 3,60 \\
\hline DERROTADO & 1,69 & 1,33 & 5,00 & 3,13 & ESCALDANTE & 4,20 & 1,89 & 3,86 & 2,76 & FERIDAS & 4,29 & 2,52 & 4,65 & 2,51 \\
\hline DESAFIANTE & 4,37 & 2,93 & 5,64 & 2,86 & ESCÂNDALO & 3,31 & 2,22 & 5,51 & 2,73 & FERIMENTO & 2,54 & 1,84 & 5,08 & 2,59 \\
\hline DESAFIO & 7,20 & 1,97 & 6,00 & 2,84 & ESCONDER & 4,08 & 1,91 & 4,51 & 2,78 & FERRAMENTA & 7,07 & 2,11 & 4,78 & 2,79 \\
\hline DESAGRADADO & 4,83 & 2,49 & 5,68 & 2,38 & ESCORBUTO & 3,77 & 1,94 & 4,03 & 2,55 & FERRO & 4,91 & 1,72 & 3,84 & 2,06 \\
\hline
\end{tabular}


Anexo 1 - Versão brasileira do

Affective Norms for English Words (ANEW-Br) (cont.)

\begin{tabular}{|c|c|c|c|c|c|c|c|c|c|c|c|c|c|c|}
\hline \multirow[b]{2}{*}{ Palavra } & \multicolumn{2}{|c|}{ Valência } & \multicolumn{2}{|c|}{ Alerta } & \multirow[b]{2}{*}{ Palavra } & \multicolumn{2}{|c|}{ Valência } & Ale & & & Valê & ncia & Ale & ta \\
\hline & Média & DP & Média & DP & & Média & DP & Média & DP & Palavra & Média & DP & Média & DP \\
\hline FESTA & 8,22 & 1,63 & 4,49 & 3,31 & HOSPITAL & 2,75 & 2,55 & 5,39 & 3,18 & LANTERNA & 5,95 & 1,56 & 3,06 & 2,21 \\
\hline FESTIVO & 8,09 & 1,76 & 5,54 & 3,31 & HOSTIL & 5,60 & 2,97 & 4,58 & 2,72 & LÁPIS & 6,24 & 1,62 & 3,42 & 2,12 \\
\hline FIRMAMENTO & 6,66 & 2,19 & 4,00 & 2,93 & HUMANITÁRIO & 4,58 & 3,69 & 5,31 & 3,34 & LARVA & 3,07 & 1,89 & 4,27 & 2,75 \\
\hline FIRME & 6,97 & 2,04 & 3,94 & 2,46 & HUMBÚRGUER & 6,91 & 2,47 & 4,19 & 2,80 & LEAL & 8,27 & 1,47 & 3,21 & 2,70 \\
\hline FLÁCIDO & 2,43 & 1,97 & 4,77 & 2,90 & HUMILDE & 7,66 & 1,88 & 3,60 & 2,69 & LEÃO & 5,89 & 2,16 & 5,51 & 2,54 \\
\hline FLEXÍVEL & 7,25 & 2,29 & 4,06 & 2,74 & HUMILHAR & 4,36 & 3,34 & 5,80 & 3,11 & LEITE & 6,68 & 2,10 & 3,01 & 2,35 \\
\hline FLORESCER & 6,38 & 2,49 & 3,31 & 2,49 & IATE & 6,73 & 1,98 & 3,97 & 2,37 & LENTO & 3,77 & 2,07 & 4,66 & 2,98 \\
\hline FOFOCA & 2,44 & 2,29 & 5,65 & 3,11 & IDÉIA & 8,14 & 1,60 & 5,31 & 3,32 & LEPRA & 1,87 & 1,46 & 4,83 & 2,99 \\
\hline FOGÃO & 4,71 & 2,75 & 4,76 & 3,02 & IDENTIDADE & 7,35 & 1,92 & 4,50 & 2,90 & LÉSBICA & 4,01 & 1,90 & 3,65 & 2,72 \\
\hline FOGO & 4,55 & 2,68 & 5,35 & 2,68 & IDIOTA & 2,28 & 2,00 & 5,28 & 2,90 & LETÁRGICO & 3,93 & 1,77 & 3,31 & 2,31 \\
\hline FORÇA & 4,62 & 2,46 & 4,76 & 2,77 & ÍDOLO & 5,99 & 2,52 & 3,73 & 2,36 & LETRA & 6,50 & 2,00 & 3,40 & 2,57 \\
\hline FORTE & 7,66 & 1,66 & 4,66 & 3,01 & IGNORÂNCIA & 2,11 & 1,83 & 5,57 & 2,99 & LIBERDADE & 8,80 & 0,57 & 5,27 & 3,73 \\
\hline FORTUITO & 6,61 & 2,07 & 3,91 & 2,60 & IGREJA & 4,22 & 2,75 & 4,70 & 2,72 & LÍDER & 6,92 & 1,96 & 4,27 & 2,89 \\
\hline FOTOGRAFIA & 7,71 & 1,96 & 4,61 & 3,15 & IMAGINAR & 8,02 & 1,69 & 5,06 & 3,25 & LIGA & 5,88 & 1,47 & 3,59 & 2,14 \\
\hline FRAGRÂNCIA & 5,98 & 2,51 & 3,80 & 2,45 & IMATURO & 2,85 & 1,88 & 5,70 & 2,66 & LINDO & 8,17 & 1,43 & 3,90 & 3,31 \\
\hline FREIRA & 4,89 & 2,49 & 3,91 & 2,62 & IMPLICAR & 4,69 & 2,80 & 5,47 & 2,63 & LIVRE & 8,37 & 1,48 & 3,95 & 3,29 \\
\hline FRÍGIDA & 2,43 & 2,07 & 4,79 & 2,78 & IMPOTENTE & 2,08 & 1,54 & 5,34 & 3,00 & LIVRO & 7,18 & 1,97 & 4,14 & 2,75 \\
\hline FRIO & 4,45 & 2,71 & 4,85 & 2,70 & IMPRESSIONADO & 6,97 & 2,42 & 4,09 & 2,82 & LIXO & 2,48 & 2,14 & 5,14 & 3,00 \\
\hline FRUSTRADO & 1,84 & 1,57 & 5,84 & 2,84 & IMUNDÍCIE & 1,86 & 1,74 & 5,18 & 2,88 & LODO & 2,91 & 2,17 & 4,41 & 2,64 \\
\hline FUGA & 3,63 & 2,39 & 5,26 & 2,82 & INCENTIVO & 4,76 & 3,25 & 5,40 & 2,67 & LOIRO & 5,97 & 1,95 & 3,37 & 2,48 \\
\hline FUNERAL & 1,52 & 1,39 & 4,89 & 3,34 & INCOMODADO & 2,45 & 1,90 & 5,66 & 2,64 & LOTERIA & 7,03 & 2,15 & 5,11 & 2,98 \\
\hline FUNGO & 3,84 & 1,99 & 4,65 & 2,56 & INCOMODAR & 2,97 & 2,22 & 5,86 & 2,79 & LOUCO & 3,87 & 2,33 & 4,90 & 2,79 \\
\hline FURACÃO & 2,72 & 2,35 & 6,08 & 2,84 & INCUMBÊNCIA & 5,02 & 2,10 & 4,29 & 2,30 & LUCRO & 7,64 & 1,86 & 5,48 & 3,07 \\
\hline GABINETE & 5,35 & 1,87 & 3,88 & 2,38 & INDIFERENTE & 4,37 & 2,37 & 4,59 & 2,24 & LUSTRE & 5,65 & 1,80 & 2,58 & 1,92 \\
\hline GANGRENA & 2,64 & 2,05 & 4,81 & 2,81 & INDÚSTRIA & 5,63 & 2,55 & 4,50 & 2,50 & LUTA & 5,66 & 2,81 & 5,51 & 2,53 \\
\hline GARFO & 6,12 & 2,03 & 3,83 & 2,47 & INFANTE & 2,99 & 2,11 & 5,24 & 2,84 & LUTO & 1,70 & 1,39 & 5,32 & 3,15 \\
\hline GAROTOS & 6,94 & 2,28 & 4,77 & 2,97 & INFECÇÃO & 1,77 & 1,54 & 5,61 & 2,96 & LUXO & 6,82 & 1,90 & 4,65 & 2,72 \\
\hline GARRAFA & 6,49 & 2,35 & 4,19 & 2,77 & INFELIZ & 1,54 & 1,37 & 6,20 & 3,01 & LUXÚRIA & 4,15 & 2,48 & 4,71 & 2,92 \\
\hline GATINHO & 7,06 & 2,72 & 3,95 & 3,13 & INFERIOR & 2,49 & 1,86 & 4,50 & 2,73 & LUZ & 7,96 & 1,64 & 4,25 & 3,02 \\
\hline GATO & 5,75 & 2,76 & 4,04 & 2,72 & INFERNO & 3,81 & 2,85 & 4,88 & 3,13 & MACHUCADO & 2,38 & 1,75 & 5,65 & 2,90 \\
\hline GATO & 3,47 & 2,60 & 5,24 & 2,76 & INFIEL & 2,08 & 1,97 & 6,34 & 3,15 & MACIO & 7,74 & 1,79 & 4,08 & 3,09 \\
\hline GELADEIRA & 6,86 & 1,72 & 3,33 & 2,61 & INOCENTE & 3,74 & 2,53 & 5,45 & 2,68 & MÃE & 8,75 & 1,14 & 3,31 & 3,20 \\
\hline GELÉIA & 5,84 & 1,96 & 3,70 & 2,22 & INSANO & 3,11 & 1,96 & 4,61 & 2,52 & MÁGICO & 7,38 & 1,83 & 4,54 & 2,91 \\
\hline GERMES & 4,69 & 2,93 & 5,46 & 2,77 & INSOSSO & 3,55 & 1,92 & 4,21 & 2,60 & MALCHEIROSO & 1,80 & 1,34 & 4,89 & 3,04 \\
\hline GINASTA & 6,40 & 1,93 & 4,12 & 2,70 & INSPIRADO & 4,72 & 3,51 & 5,48 & 3,24 & MALÍCIA & 4,47 & 2,59 & 4,43 & 2,89 \\
\hline GLAMOUR & 5,26 & 2,04 & 4,13 & 2,28 & INSPIRAR & 7,31 & 2,15 & 4,03 & 3,10 & MALUCO & 4,31 & 2,66 & 5,46 & 2,57 \\
\hline GLÓRIA & 8,16 & 1,58 & 4,72 & 3,45 & INSULTO & 5,28 & 3,52 & 4,72 & 3,32 & MALVADO & 2,09 & 1,78 & 5,77 & 2,91 \\
\hline GOLFISTA & 4,00 & 2,09 & 4,66 & 2,54 & INTELECTO & 6,80 & 2,37 & 4,33 & 2,49 & MAMILO & 5,51 & 1,37 & 4,18 & 2,58 \\
\hline GOLPE & 2,20 & 2,03 & 5,32 & 2,95 & INTELIGENTE & 7,81 & 2,28 & 4,75 & 3,26 & MANEIRA & 5,85 & 1,74 & 3,62 & 2,26 \\
\hline GORDO & 4,78 & 3,02 & 4,82 & 2,92 & INTERCURSO & 5,02 & 1,36 & 2,33 & 6,42 & MANÍACO & 1,95 & 1,74 & 6,24 & 3,03 \\
\hline GOSTO & 7,57 & 1,67 & 4,31 & 2,74 & INTERESSE & 4,74 & 3,21 & 5,68 & 2,90 & MANSO & 6,73 & 2,02 & 2,96 & 2,25 \\
\hline GRACINHA & 7,15 & 2,02 & 3,73 & 2,82 & ÍNTIMO & 7,27 & 1,88 & 4,80 & 2,84 & MANTEIGA & 5,68 & 2,11 & 3,25 & 2,30 \\
\hline GRADUADO & 8,17 & 1,69 & 5,30 & 3,37 & INTROMETER & 2,40 & 1,81 & 5,73 & 2,67 & MÃO & 6,88 & 2,07 & 2,95 & 2,42 \\
\hline GRAMA & 5,75 & 1,95 & 3,21 & 2,12 & INTROMETIDO & 2,52 & 2,00 & 5,94 & 2,56 & MÁQUINA & 5,61 & 1,77 & 3,96 & 2,50 \\
\hline GRAMADO & 7,75 & 1,85 & 3,90 & 3,18 & INTRUSO & 2,29 & 1,65 & 5,99 & 2,56 & MARAVILHA & 6,80 & 2,37 & 3,72 & 2,83 \\
\hline GRAMPOS & 6,54 & 2,37 & 3,42 & 2,49 & INUNDAÇÃO & 1,88 & 1,68 & 6,02 & 2,80 & MAREADO & 4,78 & 1,48 & 3,35 & 2,22 \\
\hline GRANADA & 2,00 & 2,05 & 6,03 & 3,16 & INÚTIL & 4,81 & 2,97 & 5,63 & 2,82 & MARICAS & 4,23 & 1,87 & 3,26 & 2,26 \\
\hline GRITO & 4,42 & 2,44 & 6,19 & 2,31 & INVASOR & 2,20 & 2,14 & 6,61 & 2,70 & MARTELO & 5,04 & 1,55 & 3,30 & 2,24 \\
\hline GROSSO & 2,71 & 1,98 & 5,88 & 2,63 & INVESTIR & 4,35 & 3,14 & 6,16 & 2,69 & MASSA & 7,13 & 2,18 & 2,99 & 2,46 \\
\hline GUERRA & 1,61 & 1,62 & 6,79 & 2,97 & IRMÃO & 7,78 & 2,15 & 5,11 & 3,00 & MASSACRE & 1,31 & 0,92 & 5,94 & 3,26 \\
\hline GUILHOTINA & 5,56 & 3,09 & 4,27 & 2,92 & IRRITAR & 4,45 & 3,02 & 4,99 & 3,15 & MASTIGAR & 6,62 & 2,01 & 3,35 & 2,50 \\
\hline GULA & 3,88 & 2,54 & 4,94 & 2,67 & ITEM & 5,08 & 1,33 & 3,13 & 2,06 & MASTURBAR & 5,60 & 2,28 & 5,21 & 2,87 \\
\hline HABILIDADE & 6,91 & 1,91 & 4,27 & 2,36 & JANELA & 7,29 & 2,13 & 3,32 & 2,71 & MATADOR & 1,49 & 1,11 & 6,69 & 3,21 \\
\hline HABITANTE & 5,55 & 1,74 & 3,34 & 2,06 & JANTA & 7,47 & 2,02 & 4,61 & 2,94 & MATERIAL & 6,07 & 1,85 & 3,37 & 2,06 \\
\hline HÁBITO & 4,13 & 2,48 & 4,95 & 2,39 & JARDIM & 5,85 & 2,80 & 4,52 & 3,03 & MEDO & 2,13 & 1,77 & 6,36 & 3,05 \\
\hline HEMODIÁLISE & 3,78 & 2,42 & 4,90 & 2,94 & JARRA & 5,72 & 1,95 & 3,66 & 2,57 & MEL & 7,28 & 2,15 & 3,48 & 2,70 \\
\hline HEROÍNA & 5,35 & 3,23 & 4,89 & 3,18 & JIBÓIA & 4,96 & 2,58 & 4,39 & 2,86 & MELHORAR & 8,21 & 1,55 & 4,58 & 3,02 \\
\hline HIDRANTE & 6,45 & 2,08 & 3,75 & 2,47 & JOGO & 6,34 & 2,35 & 4,89 & 2,66 & MELODIA & 7,73 & 1,66 & 4,51 & 3,22 \\
\hline HIDROFOBIA & 3,48 & 2,16 & 3,67 & 2,74 & JÓIA & 7,76 & 1,82 & 4,19 & 2,94 & MEMÓRIA & 6,36 & 2,33 & 4,43 & 2,75 \\
\hline HISTÓRIA & 3,54 & 3,08 & 5,48 & 3,23 & JUSTIÇA & 6,52 & 2,95 & 5,61 & 2,73 & MEMÓRIAS & 6,96 & 2,43 & 5,14 & 2,98 \\
\hline HOMEM & 6,85 & 2,78 & 4,47 & 3,16 & JUVENTUDE & 4,51 & 3,55 & 5,70 & 3,20 & MENINA & 7,10 & 1,77 & 3,26 & 2,54 \\
\hline HOMICIDA & 5,13 & 3,44 & 5,54 & 3,09 & KETCHUP & 5,31 & 2,54 & 3,92 & 2,54 & MENINO & 7,36 & 1,81 & 4,77 & 2,75 \\
\hline HONESTO & 8,60 & 1,20 & 3,32 & 5,38 & LADRÃO & 2,90 & 2,19 & 5,40 & 3,08 & MENOSPREZADO & 2,03 & 1,89 & 6,05 & 3,15 \\
\hline HONRA & 4,75 & 3,43 & 5,09 & 3,04 & LAGO & 7,38 & 2,20 & 3,82 & 2,99 & MENSAGEIRO & 6,05 & 1,58 & 3,97 & 2,21 \\
\hline HORRÍVEL & 1,98 & 1,78 & 5,55 & 2,84 & LAMA & 2,58 & 2,17 & 5,36 & 2,99 & MENTE & 7,03 & 2,01 & 3,91 & 2,85 \\
\hline HORROR & 2,54 & 2,11 & 5,83 & 2,90 & LÂMPADA & 6,51 & 2,41 & 3,82 & 2,65 & MENTIRA & 1,35 & 0,97 & 6,28 & 3,07 \\
\hline
\end{tabular}


Anexo 1 - Versão brasileira do

Affective Norms for English Words (ANEW-Br) (cont.)

\begin{tabular}{|c|c|c|c|c|c|c|c|c|c|c|c|c|c|c|}
\hline \multirow[b]{2}{*}{ Palavra } & \multicolumn{2}{|c|}{ Valência } & \multicolumn{2}{|c|}{ Alerta } & \multirow[b]{2}{*}{ Palavra } & \multicolumn{2}{|c|}{ Valência } & Ale & rta & & Valê & ncia & Ale & rta \\
\hline & Média & DP & Média & DP & & Média & DP & Média & DP & Palavra & Média & DP & Média & DP \\
\hline MERCADO & 6,59 & 2,20 & 4,12 & 2,70 & OPINIÃO & 6,78 & 2,09 & 5,21 & 2,60 & PODEROSO & 6,09 & 2,17 & 4,88 & 2,64 \\
\hline MERETRIZ & 3,82 & 1,91 & 3,52 & 2,41 & ORGASMO & 7,92 & 1,65 & 4,37 & 3,39 & PODRE & 1,81 & 1,58 & 5,10 & 3,05 \\
\hline MÊS & 6,11 & 1,90 & 3,90 & 2,51 & ORGULHOSO & 5,63 & 2,94 & 4,85 & 2,90 & POESIA & 7,66 & 1,74 & 3,13 & 2,76 \\
\hline MESA & 6,18 & 1,50 & 2,75 & 2,00 & ORQUESTRA & 7,13 & 1,92 & 4,77 & 2,88 & POLUIR & 1,51 & 1,14 & 6,05 & 2,91 \\
\hline METAL & 5,15 & 1,32 & 3,17 & 2,04 & OTIMISMO & 8,41 & 1,26 & 4,13 & 3,01 & POMBA & 6,04 & 2,37 & 2,97 & 2,25 \\
\hline MÉTODO & 6,06 & 2,13 & 3,83 & 2,59 & OURO & 6,99 & 2,15 & 4,25 & 2,91 & PORÃO & 3,86 & 1,91 & 4,22 & 2,60 \\
\hline MILIONÁRIO & 6,55 & 2,23 & 4,19 & 2,83 & OUTONO & 5,95 & 2,33 & 4,35 & 2,65 & PORTA & 5,92 & 1,95 & 3,97 & 2,49 \\
\hline MISÉRIA & 1,21 & 1,07 & 5,89 & 3,22 & OVO & 6,12 & 1,80 & 2,78 & 2,19 & PÔSTER & 6,05 & 2,12 & 3,51 & 2,34 \\
\hline MÍSTICO & 6,60 & 2,33 & 3,44 & 2,63 & PACIENTE & 5,96 & 2,64 & 4,06 & 2,77 & POVO & 6,22 & 2,27 & 4,87 & 2,46 \\
\hline MOBILIDADE & 6,17 & 1,75 & 4,06 & 2,59 & PADRE & 5,29 & 2,21 & 2,70 & 2,33 & PRADARIA & 5,77 & 1,98 & 3,51 & 2,43 \\
\hline MODESTO & 6,79 & 1,92 & 3,08 & 2,23 & PAI & 8,08 & 2,28 & 5,55 & 3,29 & PRAIA & 8,29 & 1,43 & 4,64 & 3,58 \\
\hline MOEDA & 6,76 & 2,07 & 4,10 & 2,79 & PAÍS & 5,80 & 2,56 & 4,53 & 2,76 & PRANTO & 2,52 & 2,01 & 5,49 & 2,71 \\
\hline MOFO & 2,48 & 1,98 & 4,64 & 2,89 & PAIXÃO & 6,34 & 2,71 & 5,27 & 3,30 & PRAZER & 8,65 & 1,05 & 5,29 & 3,63 \\
\hline MOINHO & 5,45 & 1,16 & 3,44 & 2,18 & PALÁCIO & 6,28 & 2,05 & 3,60 & 2,45 & PREGUIÇOSO & 3,66 & 2,31 & 4,14 & 2,65 \\
\hline MOLDE & 5,21 & 1,79 & 3,48 & 2,32 & PANFLETO & 6,28 & 2,30 & 3,45 & 2,62 & PREJUDICADO & 2,13 & 1,52 & 5,59 & 2,73 \\
\hline MONTANHA & 7,13 & 2,03 & 3,72 & 2,78 & PANQUECA & 6,88 & 1,97 & 4,03 & 2,75 & PRESENTE & 8,33 & 1,31 & 4,81 & 3,22 \\
\hline MORAL & 6,72 & 2,07 & 3,45 & 2,43 & PÂNTANO & 4,15 & 2,03 & 4,33 & 2,70 & PRESSÃO & 3,16 & 2,09 & 5,90 & 2,83 \\
\hline MÓRBIDO & 2,07 & 1,69 & 5,24 & 2,99 & PAPEL & 7,29 & 2,08 & 3,85 & 2,93 & PRESTÍGIO & 7,75 & 2,01 & 4,30 & 3,03 \\
\hline MORTE & 1,40 & 1,04 & 6,18 & 3,14 & PAQUERAR & 7,99 & 1,51 & 4,45 & 3,06 & PRETO & 5,26 & 2,19 & 3,85 & 2,35 \\
\hline MORTO & 2,01 & 1,82 & 5,11 & 3,22 & PARAÍSO & 7,07 & 2,10 & 4,79 & 3,23 & PRIMAVERA & 8,33 & 1,18 & 3,76 & 3,30 \\
\hline MOSQUITO & 2,09 & 1,71 & 5,60 & 3,10 & PARALISIA & 1,86 & 1,69 & 5,77 & 3,24 & PRIMO & 6,63 & 2,29 & 3,62 & 2,51 \\
\hline MOTIM & 3,77 & 2,25 & 4,40 & 2,88 & PARTE & 6,43 & 2,17 & 3,32 & 2,44 & PRISÃO & 1,68 & 1,39 & 5,79 & 3,00 \\
\hline MOTOR & 5,09 & 1,82 & 3,96 & 2,35 & PASSAGEM & 5,00 & 2,52 & 4,01 & 2,77 & PRIVAÇÃO & 2,86 & 2,16 & 5,53 & 2,66 \\
\hline MUCO & 5,43 & 2,35 & 3,35 & 1,98 & PÁSSARO & 7,11 & 2,06 & 4,06 & 2,76 & PRIVACIDADE & 7,99 & 1,54 & 3,73 & 2,97 \\
\hline MULETA & 2,81 & 1,94 & 4,75 & 2,77 & PATENTE & 4,77 & 1,91 & 3,23 & 2,41 & PROBLEMA & 2,17 & 1,74 & 6,14 & 2,75 \\
\hline MULHER & 6,57 & 1,90 & 3,89 & 2,60 & PATRIOTA & 6,21 & 1,97 & 3,70 & 2,70 & PROCESSO & 4,31 & 2,27 & 4,97 & 2,54 \\
\hline MUNDO & 6,17 & 2,65 & 5,01 & 2,92 & $\mathrm{PAZ}$ & 8,64 & 1,14 & 3,71 & 3,50 & PROEMINENTE & 5,43 & 1,58 & 4,10 & 2,21 \\
\hline MUSCULAR & 6,99 & 2,55 & 4,54 & 3,27 & PAZINHA & 4,41 & 1,73 & 3,76 & 2,47 & PROFESSOR & 6,74 & 1,95 & 4,54 & 2,51 \\
\hline MUSEU & 6,01 & 2,01 & 3,00 & 2,13 & PÉ & 5,99 & 2,40 & 2,91 & 2,48 & PROGRESSO & 7,90 & 1,89 & 4,76 & 3,09 \\
\hline MÚSICA & 5,18 & 3,70 & 5,73 & 3,31 & PECADO & 3,29 & 2,14 & 4,23 & 2,82 & PROMOÇÃO & 7,89 & 1,88 & 4,90 & 3,07 \\
\hline MUTAÇÃO & 4,53 & 2,26 & 4,40 & 2,75 & PECAMINOSO & 3,43 & 2,09 & 4,72 & 2,74 & PRÓSPERO & 7,78 & 1,92 & 4,44 & 3,11 \\
\hline MUTILAR & 4,66 & 3,57 & 5,46 & 3,33 & PEÇONHA & 4,67 & 2,28 & 4,33 & 2,56 & PROSTITUTA & 2,58 & 2,03 & 4,55 & 2,98 \\
\hline NADADOR & 6,38 & 2,16 & 3,49 & 2,82 & PEDINTE & 3,23 & 2,11 & 4,85 & 2,58 & PROTEGIDO & 7,26 & 2,21 & 3,45 & 2,70 \\
\hline NATAL & 8,06 & 1,98 & 3,69 & 3,11 & PÊLO & 4,29 & 1,80 & 3,65 & 2,30 & PUNIÇÃO & 2,89 & 2,17 & 5,44 & 2,88 \\
\hline NATURAL & 4,97 & 3,36 & 4,76 & 3,16 & PENALIDADE & 5,43 & 2,61 & 4,86 & 2,87 & PUS & 2,74 & 1,85 & 4,77 & 2,73 \\
\hline NATUREZA & 8,58 & 1,09 & 3,25 & 3,03 & PENHASCO & 3,43 & 2,22 & 5,21 & 3,08 & PÚTRIDO & 3,25 & 2,07 & 4,16 & 2,59 \\
\hline NÁUSEA & 3,95 & 2,72 & 4,78 & 2,77 & PÊNIS & 6,85 & 1,78 & 5,03 & 2,89 & QUADRADO & 5,18 & 1,95 & 2,95 & 1,97 \\
\hline NAVALHA & 2,64 & 1,81 & 5,00 & 3,24 & PENITENTE & 4,06 & 1,88 & 4,01 & 2,55 & QUADRO & 5,87 & 1,85 & 3,80 & 2,22 \\
\hline NAVIO & 6,55 & 2,16 & 3,68 & 2,72 & PENSAMENTO & 4,88 & 3,09 & 5,00 & 2,82 & QUALIDADE & 8,29 & 1,43 & 4,61 & 3,20 \\
\hline NECROTÉRIO & 2,10 & 2,07 & 5,75 & 3,15 & PENSATIVO & 5,99 & 2,23 & 4,36 & 2,75 & QUEBRADO & 2,57 & 1,78 & 5,23 & 2,64 \\
\hline NÉCTAR & 4,66 & 3,08 & 5,41 & 3,25 & PERDEDOR & 4,22 & 3,02 & 5,54 & 2,97 & QUEDA & 2,60 & 2,00 & 5,84 & 2,59 \\
\hline NEGLIGÊNCIA & 2,40 & 1,80 & 5,78 & 2,85 & PERDIDO & 2,93 & 1,67 & 5,17 & 2,55 & QUEIMADURA & 1,94 & 1,58 & 5,54 & 3,05 \\
\hline NERVOSO & 4,21 & 3,04 & 5,33 & 3,05 & PERFEIÇÃO & 6,85 & 2,30 & 4,87 & 2,84 & QUEIXO & 5,79 & 1,56 & 2,97 & 2,02 \\
\hline NEURÓTICO & 2,49 & 1,65 & 6,10 & 2,98 & PERFUME & 8,20 & 1,24 & 4,33 & 3,31 & QUERIDO & 8,36 & 1,20 & 4,01 & 3,16 \\
\hline NEVE & 6,93 & 2,27 & 5,19 & 2,91 & PERIGO & 2,56 & 2,06 & 6,74 & 2,47 & QUEROSENE & 4,04 & 1,87 & 3,78 & 2,53 \\
\hline NÓ & 3,90 & 1,79 & 4,76 & 2,77 & PERSEGUIR & 3,46 & 2,26 & 5,50 & 2,63 & QUIETO & 5,23 & 2,02 & 3,21 & 2,15 \\
\hline NOIVA & 6,84 & 1,90 & 5,31 & 2,78 & PERTURBADO & 2,49 & 1,57 & 6,01 & 2,71 & QUIMIOTERAPIA & 1,97 & 1,91 & 5,61 & 3,18 \\
\hline NOME & 7,50 & 1,98 & 3,53 & 2,79 & PERTURBAR & 2,32 & 1,58 & 5,35 & 2,96 & $\mathrm{R} \tilde{\mathrm{A}}$ & 4,01 & 2,05 & 4,11 & 2,87 \\
\hline NOTÍCIA & 6,49 & 1,77 & 4,93 & 2,62 & PERVERTIDO & 3,35 & 2,32 & 5,09 & 2,72 & RADIADOR & 4,75 & 1,95 & 3,63 & 2,31 \\
\hline NOVO & 7,96 & 1,31 & 4,94 & 2,94 & PESADELO & 2,11 & 1,88 & 5,92 & 3,11 & RADIANTE & 8,08 & 1,61 & 4,70 & 3,28 \\
\hline NU & 7,14 & 1,65 & 4,49 & 2,88 & PESAR & 3,41 & 2,40 & 4,78 & 2,60 & RÁDIO & 7,84 & 1,70 & 4,12 & 3,13 \\
\hline NUBLADO & 3,91 & 2,06 & 3,73 & 2,53 & PESSOA & 7,85 & 1,61 & 4,46 & 2,98 & RAINHA & 6,27 & 1,95 & 3,99 & 2,52 \\
\hline NUTRIR & 6,42 & 2,36 & 4,90 & 2,72 & PESTE & 2,17 & 1,67 & 5,43 & 2,84 & RAIVA & 1,97 & 1,72 & 7,13 & 2,49 \\
\hline NUVEM & 6,67 & 2,07 & 2,70 & 2,16 & PIADA & 8,14 & 1,30 & 4,45 & 3,07 & RALÉ & 2,94 & 1,81 & 3,96 & 2,36 \\
\hline OBEDECER & 4,55 & 2,46 & 4,20 & 2,62 & PICADA & 2,58 & 1,53 & 5,12 & 2,53 & RANÇOSO & 2,49 & 1,82 & 5,16 & 2,57 \\
\hline OBESIDADE & 2,14 & 1,99 & 5,55 & 3,30 & PIEDADE & 5,82 & 2,21 & 4,08 & 2,53 & RÁPIDO & 6,32 & 2,23 & 5,37 & 2,64 \\
\hline OBSCENO & 3,89 & 1,98 & 4,09 & 2,77 & PINTAR & 7,26 & 1,88 & 3,68 & 2,80 & RATO & 3,19 & 2,09 & 5,18 & 2,87 \\
\hline OBSESSÃO & 3,00 & 2,07 & 5,19 & 2,86 & PIOLHO & 2,08 & 1,52 & 5,08 & 3,17 & RAZÃO & 6,49 & 2,25 & 4,56 & 2,40 \\
\hline OBSTRUIR & 2,64 & 1,78 & 5,29 & 3,00 & PISCAR & 6,38 & 1,86 & 3,26 & 2,38 & REALIZAÇÃO & 8,51 & 1,07 & 5,23 & 3,47 \\
\hline OCEANO & 7,77 & 1,81 & 3,58 & 2,93 & PISTOLA & 2,18 & 1,82 & 5,83 & 3,03 & RECEOSO & 3,89 & 1,86 & 4,51 & 2,37 \\
\hline ÓDIO & 2,86 & 2,16 & 4,76 & 3,05 & PIZZA & 8,35 & 1,18 & 4,16 & 3,30 & RECOMPENSA & 7,59 & 2,16 & 4,96 & 3,05 \\
\hline OFENDER & 1,78 & 1,48 & 6,23 & 3,14 & PLANÍCIE & 6,24 & 1,95 & 3,17 & 2,43 & RECREIO & 7,65 & 1,86 & 4,10 & 2,96 \\
\hline OFUSCAR & 4,51 & 2,04 & 4,14 & 2,47 & PLANO & 7,11 & 1,91 & 4,96 & 2,75 & REFÉM & 1,84 & 1,55 & 6,55 & 2,61 \\
\hline ÔNIBUS & 4,18 & 2,39 & 4,58 & 2,91 & PLANTA & 7,83 & 1,53 & 3,22 & 2,74 & REFRESCO & 7,86 & 1,64 & 3,38 & 2,91 \\
\hline ONIPOTENTE & 5,80 & 2,63 & 4,76 & 2,81 & POBREZA & 1,41 & 1,18 & 5,83 & 2,79 & REI & 5,73 & 2,22 & 3,71 & 2,46 \\
\hline OPÇÃO & 6,27 & 1,85 & 5,18 & 2,86 & PODER & 5,53 & 2,58 & 5,39 & 2,63 & REJEITADO & 1,61 & 1,24 & 5,85 & 2,73 \\
\hline
\end{tabular}


Anexo 1 - Versão brasileira do

Affective Norms for English Words (ANEW-Br) (cont.)

\begin{tabular}{|c|c|c|c|c|c|c|c|c|c|c|c|c|c|c|}
\hline \multirow[b]{2}{*}{ Palavra } & \multicolumn{2}{|c|}{ Valência } & \multicolumn{2}{|c|}{ Alerta } & \multirow[b]{2}{*}{ Palavra } & \multicolumn{2}{|c|}{ Valência } & \multicolumn{2}{|c|}{ Alerta } & \multirow[b]{2}{*}{ Palavra } & \multicolumn{2}{|c|}{ Valência } & \multicolumn{2}{|c|}{ Alerta } \\
\hline & Média & DP & Média & DP & & Média & DP & Média & DP & & Média & DP & Média & DP \\
\hline RELÂMPAGO & 3,77 & 2,31 & 5,85 & 2,72 & SOLIDÃO & 1,58 & 1,28 & 5,37 & 2,82 & TRAVESSURA & 6,56 & 1,87 & 4,84 & 2,52 \\
\hline RELAXADO & 6,36 & 3,18 & 3,76 & 3,01 & SOLITÁRIO & 2,47 & 2,09 & 5,28 & 2,95 & TREVAS & 1,74 & 1,46 & 5,46 & 2,99 \\
\hline RELÓGIO & 5,73 & 2,17 & 4,42 & 2,35 & SOMBRA & 6,19 & 2,15 & 3,49 & 2,38 & TRISTE & 1,73 & 1,29 & 5,28 & 2,80 \\
\hline REMÉDIO & 3,93 & 2,58 & 4,24 & 2,54 & SOMBRINHA & 5,09 & 2,09 & 3,78 & 2,32 & TRIUNFANTE & 8,14 & 1,42 & 4,99 & 3,24 \\
\hline RÉPTIL & 4,47 & 2,18 & 4,34 & 2,59 & SONHO & 7,59 & 1,93 & 4,36 & 3,03 & TRIUNFO & 7,93 & 1,52 & 4,63 & 3,01 \\
\hline REPUGNADO & 2,67 & 1,80 & 4,56 & 2,31 & SONO & 6,10 & 2,63 & 3,22 & 2,74 & TROFÉU & 8,04 & 1,65 & 4,71 & 3,40 \\
\hline REPULSIVO & 3,10 & 2,15 & 5,15 & 2,35 & SÓRDIDO & 2,90 & 2,10 & 4,55 & 2,57 & TROMPETE & 5,84 & 2,04 & 3,54 & 2,49 \\
\hline RESERVADO & 5,75 & 1,78 & 3,76 & 2,26 & SORRISO & 8,50 & 1,33 & 4,15 & 3,49 & TRONCO & 5,44 & 1,77 & 2,93 & 2,16 \\
\hline RESPEITO & 8,43 & 1,06 & 3,90 & 3,12 & SORTUDO & 7,46 & 2,01 & 4,51 & 2,96 & TUBARÃO & 3,40 & 2,19 & 6,21 & 2,64 \\
\hline RESPEITOSO & 7,52 & 1,71 & 3,41 & 2,63 & SOZINHO & 2,47 & 2,04 & 4,96 & 3,03 & TUMOR & 1,44 & 1,23 & 6,29 & 3,10 \\
\hline RESPOSTA & 6,96 & 1,81 & 4,47 & 2,73 & SUAVE & 7,79 & 1,59 & 3,23 & 2,84 & TÚMULO & 2,06 & 1,84 & 5,39 & 2,87 \\
\hline RESSENTIDO & 2,83 & 1,87 & 4,80 & 2,24 & SUBJUGADO & 2,95 & 1,99 & 5,36 & 2,69 & ÚLCERA & 1,54 & 1,04 & 6,00 & 3,06 \\
\hline RESTAURANTE & 7,70 & 1,56 & 3,96 & 2,77 & SUCESSO & 8,25 & 1,51 & 5,31 & 3,18 & ULTRAJE & 3,71 & 1,78 & 4,22 & 2,39 \\
\hline REUNIÃO & 5,06 & 2,26 & 4,88 & 2,76 & SUFOCAR & 2,05 & 1,85 & 6,61 & 2,59 & UNIDADE & 5,76 & 2,26 & 2,94 & 2,48 \\
\hline REVERENTE & 5,41 & 1,86 & 3,75 & 2,08 & SUICÍDIO & 1,35 & 1,44 & 5,13 & 3,38 & UNTENSÍLIO & 6,63 & 1,60 & 3,01 & 2,32 \\
\hline REVISTA & 6,90 & 1,80 & 3,51 & 2,54 & SUJEIRA & 1,90 & 1,83 & 5,86 & 2,82 & URINA & 5,38 & 2,21 & 3,14 & 2,61 \\
\hline REVOLTA & 2,44 & 2,09 & 6,47 & 2,52 & SUJO & 2,43 & 1,99 & 5,49 & 2,75 & ÚTIL & 8,02 & 1,41 & 3,71 & 2,89 \\
\hline REVÓLVER & 1,94 & 1,78 & 6,24 & 3,05 & SURPRESO & 6,60 & 2,15 & 5,36 & 2,49 & VACA & 6,29 & 2,12 & 2,61 & 2,17 \\
\hline RICO & 6,49 & 1,89 & 4,21 & 2,71 & SURRA & 1,94 & 1,95 & 5,55 & 3,12 & VAGÃO & 4,97 & 1,72 & 3,81 & 2,55 \\
\hline RIDÍCULO & 2,61 & 1,90 & 4,87 & 2,70 & SUSPEITO & 3,14 & 2,08 & 5,64 & 2,49 & VAGINA & 6,80 & 2,02 & 4,06 & 3,08 \\
\hline RIFLE & 2,36 & 2,11 & 5,68 & 2,91 & TABACO & 2,04 & 1,86 & 5,35 & 3,18 & VAIDADE & 6,18 & 2,13 & 4,55 & 2,42 \\
\hline RÍGIDO & 4,56 & 1,89 & 4,77 & 2,14 & TALENTO & 8,31 & 1,20 & 5,04 & 3,29 & VAMPIRO & 3,55 & 2,06 & 4,38 & 2,92 \\
\hline RIO & 6,94 & 2,09 & 3,92 & 2,67 & TANQUE & 4,64 & 2,27 & 3,74 & 2,31 & VÂNDALO & 1,76 & 1,32 & 6,28 & 2,89 \\
\hline RIQUEZAS & 6,75 & 2,06 & 4,24 & 2,59 & TAPA & 2,22 & 1,91 & 6,19 & 2,59 & VANTAGEM & 5,71 & 4,08 & 4,45 & 2,62 \\
\hline RISADA & 8,59 & 0,92 & 4,63 & 3,54 & TÁXI & 5,10 & 2,08 & 3,71 & 2,39 & VARA & 3,24 & 1,27 & 3,66 & 2,50 \\
\hline ROCHA & 5,49 & 1,85 & 3,52 & 2,11 & TECIDO & 5,97 & 1,91 & 3,37 & 1,88 & VARÍOLA & 1,95 & 1,36 & 4,95 & 3,05 \\
\hline RODOVIA & 5,46 & 1,95 & 4,65 & 2,34 & TÉDIO & 2,69 & 2,01 & 5,32 & 2,60 & VEÍCULO & 7,41 & 1,73 & 4,45 & 2,61 \\
\hline ROMÂNTICO & 8,34 & 1,35 & 4,16 & 3,33 & TELEVISÃO & 6,69 & 2,21 & 4,21 & 2,76 & VELEIRO & 6,57 & 1,99 & 3,39 & 2,81 \\
\hline ROSTO & 7,53 & 1,71 & 3,95 & 2,80 & TEMIDO & 4,23 & 2,02 & 4,72 & 2,23 & VELOZ & 6,23 & 2,06 & 5,18 & 2,66 \\
\hline ROUPA & 7,41 & 2,00 & 4,57 & 3,20 & TEMÍVEL & 2,95 & 2,13 & 5,72 & 2,44 & VENCER & 8,55 & 1,35 & 5,46 & 3,40 \\
\hline RUA & 6,19 & 1,56 & 4,42 & 2,38 & TEMPERAMENTAL & 4,37 & 1,89 & 4,94 & 2,16 & VENENO & 1,54 & 1,01 & 6,77 & 2,59 \\
\hline RUDE & 2,96 & 1,82 & 5,20 & 2,34 & TEMPESTADE & 3,49 & 2,44 & 5,53 & 2,84 & VENTILADOR & 6,73 & 1,98 & 2,76 & 2,32 \\
\hline RUIDOSO & 3,97 & 1,92 & 4,80 & 2,37 & TEMPO & 5,66 & 2,20 & 5,25 & 2,43 & VERDADE & 8,56 & 1,30 & 3,46 & 3,08 \\
\hline SÁBIO & 7,92 & 1,38 & 3,96 & 2,83 & TÊNIS & 6,49 & 1,86 & 2,83 & 2,13 & VERDE & 7,39 & 2,00 & 3,26 & 2,90 \\
\hline SABOROSO & 8,24 & 1,42 & 4,05 & 3,17 & TENSO & 2,52 & 1,61 & 6,63 & 2,30 & VERMELHO & 5,84 & 2,63 & 4,94 & 2,85 \\
\hline SAFIRA & 6,00 & 1,93 & 3,40 & 2,37 & TEORIA & 5,65 & 1,93 & 3,45 & 2,28 & VESPA & 3,63 & 2,18 & 4,50 & 3,08 \\
\hline SALADA & 6,88 & 2,26 & 3,67 & 2,86 & TERMÔMETRO & 4,49 & 1,72 & 3,33 & 2,04 & VESTIBULAR & 4,93 & 2,94 & 6,65 & 2,44 \\
\hline SALVADOR & 7,67 & 1,77 & 3,57 & 2,61 & TERRA & 7,76 & 1,93 & 4,09 & 2,92 & VESTIDO & 6,69 & 2,05 & 3,63 & 2,68 \\
\hline SALVAR & 8,10 & 1,36 & 4,56 & 3,16 & TERRÍVEL & 2,12 & 1,68 & 6,41 & 2,48 & VESTUÁRIO & 6,80 & 1,84 & 3,91 & 2,70 \\
\hline SANGRENTO & 2,29 & 1,96 & 5,42 & 3,01 & TERRORISTA & 1,45 & 1,15 & 6,35 & 2,88 & VIAGEM & 8,55 & 1,22 & 5,26 & 3,39 \\
\hline SANTO & 6,65 & 2,01 & 3,44 & 2,59 & TESOURA & 4,62 & 1,72 & 3,25 & 2,34 & VIBRAÇÃO & 6,96 & 1,95 & 5,76 & 2,86 \\
\hline SARAMPO & 2,49 & 1,61 & 4,46 & 2,74 & TESOURO & 7,60 & 1,92 & 4,61 & 3,16 & VICIADO & 1,46 & 1,23 & 5,73 & 2,99 \\
\hline SATISFEITO & 8,09 & 1,58 & 3,99 & 3,04 & TÍMIDO & 4,12 & 2,30 & 4,78 & 2,66 & vÍCIO & 2,16 & 1,72 & 5,82 & 2,93 \\
\hline SAUDAR & 8,00 & 1,32 & 3,64 & 2,84 & TINTA & 6,18 & 1,68 & 2,78 & 2,36 & VIDA & 8,63 & 1,01 & 5,45 & 3,53 \\
\hline SAÚDE & 8,39 & 1,25 & 4,10 & 3,16 & TIO & 6,90 & 2,21 & 2,82 & 2,37 & VIDRO & 4,96 & 1,37 & 3,49 & 2,13 \\
\hline SECADOR & 5,61 & 1,92 & 3,96 & 2,46 & TOBOGÃ & 4,88 & 3,03 & 5,59 & 2,93 & VIGIAR & 4,13 & 2,07 & 4,91 & 2,83 \\
\hline SEDA & 6,38 & 1,94 & 2,79 & 2,34 & TOLO & 2,71 & 1,62 & 4,38 & 2,61 & VIGOROSO & 6,85 & 1,83 & 4,03 & 2,36 \\
\hline SEGURO & 7,06 & 2,46 & 3,70 & 2,69 & TORNADO & 2,41 & 1,73 & 5,89 & 2,80 & VILA & 4,94 & 2,20 & 3,89 & 2,50 \\
\hline SENTIMENTO & 7,41 & 2,02 & 4,85 & 2,85 & TORNOZELO & 5,49 & 1,75 & 2,84 & 2,35 & VINHO & 7,19 & 2,00 & 4,09 & 2,94 \\
\hline SÉRIO & 5,39 & 2,16 & 4,01 & 2,17 & TORRE & 5,51 & 1,96 & 3,82 & 2,56 & VIOLENTO & 1,46 & 1,10 & 6,49 & 2,95 \\
\hline SEVERO & 3,29 & 2,19 & 5,12 & 2,54 & TORTA & 7,71 & 1,76 & 3,92 & 2,98 & VIOLINO & 6,42 & 2,34 & 3,14 & 2,82 \\
\hline SEXO & 7,93 & 1,86 & 5,28 & 3,40 & TORTURA & 1,32 & 0,90 & 7,35 & 2,37 & VIRGEM & 5,84 & 1,99 & 3,09 & 2,52 \\
\hline SEXY & 7,66 & 1,70 & 5,26 & 2,99 & TÓXICO & 1,80 & 1,68 & 5,53 & 2,85 & VIRTUDE & 8,02 & 1,63 & 3,65 & 2,85 \\
\hline SÍFILIS & 1,93 & 1,74 & 5,37 & 3,22 & TRAGÉDIA & 1,38 & 0,98 & 7,09 & 2,36 & VISÃO & 7,33 & 2,32 & 3,89 & 2,87 \\
\hline SOBRECARREGADO & 2,58 & 1,95 & 6,49 & 2,54 & TRAIDOR & 1,29 & 1,09 & 7,16 & 2,69 & VÍTIMA & 2,26 & 1,60 & 6,08 & 2,69 \\
\hline SOBRESSALTO & 4,76 & 2,28 & 4,84 & 2,50 & TRAIR & 1,40 & 1,12 & 7,24 & 2,59 & VITÓRIA & 8,74 & 0,67 & 5,36 & 3,45 \\
\hline SOCIAL & 6,74 & 2,03 & 4,09 & 2,50 & TRANQUILAMENTE & 7,89 & 1,88 & 2,49 & 2,66 & VÍVIDO & 7,02 & 1,91 & 3,92 & 2,70 \\
\hline SOCIEDADE & 5,53 & 2,46 & 5,25 & 2,29 & TRANQÜILO & 8,07 & 1,75 & 2,22 & 2,26 & VIVO & 8,34 & 1,50 & 4,70 & 3,21 \\
\hline SOFRIMENTO & 1,68 & 1,65 & 6,14 & 2,90 & TRATAR & 6,55 & 2,06 & 3,31 & 2,31 & VÔMITO & 2,31 & 1,50 & 4,47 & 2,48 \\
\hline SOL & 8,28 & 1,68 & 4,27 & 3,38 & TRAUMA & 1,86 & 1,40 & 6,43 & 2,61 & VULCÃO & 3,74 & 2,45 & 5,50 & 2,72 \\
\hline SOLENE & 5,29 & 1,99 & 4,00 & 2,08 & TRAVESSEIRO & 8,19 & 1,56 & 2,58 & 2,80 & & & & & \\
\hline
\end{tabular}

